\title{
Methodology for Satellite Formation-Keeping in the Presence of System Uncertainties
}

\author{
Firdaus E. Udwadia* \\ University of Southern California, Los Angeles, California 90089-1453 \\ Thanapat Wanichanon $\ddagger$ \\ Mahidol University, Puttamonthon, Nakorn Pathom 73170, Thailand \\ and \\ Hancheol Cho \\ Samsung Techwin Company, Limited, Gyeonggi-do 463-400, Republic of Korea \\ DOI: $10.2514 / 1 . G 000317$
}

\begin{abstract}
A two-step formation-keeping control methodology is proposed that includes both attitude and orbital control requirements in the presence of uncertainties. Based on a nominal system model that provides the best assessment of the real-life uncertain environment, a nonlinear controller that satisfies the required attitude and orbital requirements is first developed. This controller allows the nonlinear nominal system to exactly track the desired attitude and orbital requirements without making any linearizations/approximations. In the second step, a new additional set of closed-form additive continuous controllers is developed. These continuous controllers compensate for uncertainties in the physical model of the satellite and in the forces to which it may be subjected. They obviate the problem of chattering. The desired trajectory of the nominal system is used as the tracking signal, and these controllers are based on a generalization of the concept of sliding surfaces. Error bounds on tracking due to the presence of uncertainties are analytically obtained. The resulting closed-form methodology permits the desired attitude and orbital requirements of the nominal system to be met within user-specified bounds in the presence of unknown, but bounded, uncertainties. Numerical results are provided, showing the simplicity and efficacy of the control methodology, and the reliability of the analytically obtained error bounds.
\end{abstract}

\section{Introduction}

$\mathbf{T}$ HE use of small multiple satellites flying in formation holds out the potential for advantages like reducing total mission costs, performing certain missions more flexibly and efficiently, and making possible advanced applications such as space interferometry and high-resolution imaging [1]. This paper addresses the formationkeeping problem in the presence of model uncertainties. A satellite formation is considered, in which a set of follower satellites follows, in a desired manner, a leader satellite. The aim is to develop a control methodology so that each follower satellite in the formation achieves a desired attitude and a desired formation configuration in the presence of uncertainties.

Because formation keeping is important to successfully achieve certain mission goals, numerous researchers have been attracted to this problem. However, this problem has been mostly handled by considering linear approximations of the nonlinear multiple-satellite system, and usually assuming that there are no uncertainties in defining the modeled system. Traditionally, the problem has been solved using linear control theories based on linearized equations of relative motion, such as a Hill-Clohessy-Wiltshire (HCW) equation $[2,3]$ for a circular leader satellite's orbit, or a Tschauner-Hempel equation [4] for an elliptical leader satellite's orbit. Yan et al. [5] designed a linear quadratic regulator for the satellites' periodic motion based on the HCW equation. Won and Ahn [] $]$ assumed an

Received 8 October 2013; accepted for publication 26 November 2013; published online 8 April 2014. Copyright $@ 2013$ by the American Institute of Aeronautics and Astronautics, Inc. All rights reserved. Copies of this paper may be made for personal or internal use, on condition that the copier pay the $\$ 10.00$ per-copy fee to the Copyright Clearance Center, Inc., 222 Rosewood Drive, Danvers, MA 01923; include the code 1533-3884/14 and $\$ 10.00$ in correspondence with the CCC.

*Professor, Departments of Aerospace and Mechanical Engineering, Civil Engineering, Mathematics, and Information and Operations Management, 430K Olin Hall; fudwadia@usc.edu.

'Lecturer, Department of Mechanical Engineering; thanapat.wan@ mahidol.ac.th.

${ }^{\sharp}$ Senior Research Engineer, Subsystem Group, Power Systems R\&D Center; hancheol.cho@gmail.com. elliptical leader satellite's orbit to develop the state-dependentRiccati-equation control technique for formation keeping with constant separation distance between satellites. In [7], a controller that uses Lyapunov control theory was introduced for target tracking while countering the effect of gravity-gradient torques. Ahn and Kim [8] assumed a formation of satellites as a virtual rigid-body structure to develop an algorithm for pointing to a specified target. They combined an adaptive control scheme and a sliding-mode control scheme to make the satellites follow the desired position and attitude command, considering the mass variation of the satellite and the unknown constant disturbance force and torque as the uncertainties. The controller employed uses discontinuous functions that, in general, can cause chattering while tracking the reference trajectories. Lee and Singh [9] consider variable structure model reference control, and address only the orbital motion problem related to satellite formation keeping. Godard and Kumar [10] consider the satellite formation-keeping problem, and use sliding-mode control with the leader satellite assumed to have an elliptic orbital motion. They use the linearized motion, and the controller that compensates for the uncertainties is discontinuous. The full body problem is not considered.

In this paper, a nonlinear controller obtained from the fundamental equation of mechanics [11-13] is employed that captures all the nonlinearities in the dynamic system of multiple satellites. Because of its remarkable simplicity and applicability, several researchers have used it in fields like robotics [14], modeling of complex multibody systems $[15,16]$, and also in solving the formationkeeping problem. Cho and Yu [17] obtained an analytical solution for formation keeping when the leader satellite is in an unperturbed circular orbit. They did not, however, consider any attitude dynamics. Udwadia et al. [18] solved the precision tumbling and precisiontracking problem for a nonlinear, nonautonomous multibody system. They used quaternions and included attitude dynamics; however, they did not consider any uncertainties in their dynamic model. Recently, Cho and Udwadia [19] gave an exact solution to the orbital and attitude control of a satellite formation, in which the leader satellite moves in a $J_{2}$ gravity field. In the present paper, a general methodology is developed for formation keeping, in which the 
follower satellites are required to satisfy both attitude and orbital requirements while their dynamic models are uncertain.

The control methodology is developed in two steps. The first step uses the concept of the fundamental equation to provide the closedform control force and torque needed to exactly track the attitude and orbital requirements, for the nominal system model of each satellite. The nominal model is the model adduced from the best assessment of the characteristics of the real-life system. No approximations/ linearizations in the nonlinear dynamic description of the system are made, and the nonlinear controller obtained minimizes the norm of the control force at each instant in time. In the second step of the control methodology, this nonlinear controller is augmented by an additional additive controller based on a generalization of the notion of sliding surfaces. This additive control approach is again developed in closed form, and its formulation makes available a set of continuous controllers that can accommodate the practical requirements imposed on the control effort while also eliminating the presence of chattering - a ubiquitous consequence of using conventional sliding-mode control. The method developed herein also guarantees a prespecified error bound on the tracking of the uncertain system. Standard sliding-mode control cannot provide these aforementioned advantages. The tracking-control objective having been exactly met for the nominal system allows the additional controller developed in the second step to be more efficacious and fine-tunable in taking care of the uncertainties in the actual system's description. This then provides a simple, general approach to the control of the uncertain satellite system, leading to a set of closedform nonlinear controllers that satisfies the desired attitude and orbital requirements within desired error bounds.

In contrast with the approaches developed hereto, 1) the methodology developed here deals with the full body problem, and general time-varying attitude and orbital requirements can be used; 2 ) it is general enough that it can be used for any dynamic spacecraftformation-flight system, and the leader satellite can have any prescribed orbit; 3) it develops, in closed form, a controller that minimizes the control cost at every instant of time without making any linearizations/approximation, and that can be easily implemented in real time; 4) it uses generalized sliding surfaces, thereby eliminating chattering and permitting the use of parameterized sets of continuous controllers that can accommodate the limitations of practical controllers; and 5) it provides explicit analytical error bounds on tracking performance in the presence of uncertainties. A numerical example showing the simplicity and efficacy of the approach, and the reliability of the analytical error bounds is provided.

To illustrate the methodology, a leader satellite is considered that follows a prescribed unperturbed circular orbit, with the follower satellites required to 1) circle the leader in the Hill frame, and 2) simultaneously point to a specific spot in space, which is chosen, for simplicity, as the center of Earth. The satellites are modeled as rigid bodies, and for describing the attitude dynamics, quaternions are used so that arbitrary attitude requirements and orientations can be realized while avoiding singularities. It is assumed that six actuators be equipped with each satellite: three actuators are for orbital control along each axis, and the other three are for attitude control. The general methodology is exhibited by considering uncertainties in the masses and moments of inertia of the follower satellites, because they are often difficult to exactly assess, and can change during the course of time-extended missions. Using suitable generalized coordinates, it is shown that both attitude and orbital control can be handled in a simple and unified way. Numerical results are obtained to demonstrate the accuracy of the control approach in maintaining the desired formation requirements.

\section{Description of Constrained Mechanical Systems}

The general approach that shall be followed is to view the trackingcontrol problem in the framework of constrained motion. Attitude and orbital requirements will be viewed as constraints on the nonlinear dynamic system, and explicit closed-form generalized control forces to exactly satisfy these requirements are obtained. In what follows, therefore, the terms requirements and constraints, the terms control forces and constraint forces, and the terms controlled motion and constrained motion will be interchangeably used.

As stated earlier, the best assessment of the actual real-life system will be denoted as the nominal system, that is, the best deterministic model of the system at hand. A three-step approach is followed. First, the so-called uncontrolled (unconstrained) system is described, in which the coordinates are all assumed to be independent of each other. The equation of motion of this system is given, using Lagrange's equation, by

$$
\mathbf{M}(\boldsymbol{q}, t) \ddot{\boldsymbol{q}}=\boldsymbol{Q}(\boldsymbol{q}, \dot{\boldsymbol{q}}, t)
$$

with the initial conditions

$$
\boldsymbol{q}(t=0)=\boldsymbol{q}_{0}, \quad \dot{\boldsymbol{q}}(t=0)=\dot{\boldsymbol{q}}_{0}
$$

in which $\boldsymbol{q}$ is the generalized coordinate $n$-vector and $t$ is time; $\mathbf{M}>0$ is the $n \times n$ mass matrix, which is a function of $\boldsymbol{q}$ and $t$; and $\boldsymbol{Q}$ is an $n$ vector, called the given force, which is a known function of $\boldsymbol{q}, \dot{\boldsymbol{q}}$, and $t$. From Eq. (1), one can find the acceleration of the uncontrolled system given by

$$
\boldsymbol{a}:=\mathbf{M}^{-1}(\boldsymbol{q}, t) \boldsymbol{Q}(\boldsymbol{q}, \dot{\boldsymbol{q}}, t)
$$

Second, a set of control requirements (or trajectory/orientation requirements) is imposed as constraints on this uncontrolled system. The uncontrolled system is now subjected to the $p$ constraints given by

$$
\varphi_{i}(\boldsymbol{q}, \dot{\boldsymbol{q}}, t)=0, \quad i=1,2, \ldots, p
$$

in which $r(\leq p)$ equations among $\varphi_{1}, \varphi_{2}, \ldots, \varphi_{p}$ in Eq. (4) are functionally independent. The constraints described by Eq. (4) include all the usual varieties of holonomic and/or nonholonomic constraints. It is further assumed that the set of trajectory requirements given by Eq. (4) is smooth enough so that one can differentiate them with respect to time $t$ to obtain the relation

$$
\mathbf{A}(\boldsymbol{q}, \dot{\boldsymbol{q}}, t) \ddot{\boldsymbol{q}}=\boldsymbol{b}(\boldsymbol{q}, \dot{\boldsymbol{q}}, t)
$$

in which $\mathbf{A}$ is a $p \times n$ matrix whose rank is $r$, and $\boldsymbol{b}$ is a $p$-vector. It is noted that each row of $\mathbf{A}$ arises by appropriately differentiating one of the $p$ constraint equations in the set given in Eq. (4).

Finally, the description of motion of the controlled nominal system, or the nominal system for short, is obtained as

$$
\mathbf{M}(\boldsymbol{q}, t) \ddot{\boldsymbol{q}}=\boldsymbol{Q}(\boldsymbol{q}, \dot{\boldsymbol{q}}, t)+\boldsymbol{Q}^{c}(\boldsymbol{q}, \dot{\boldsymbol{q}}, t)
$$

in which $Q^{c}$ is the control force $n$-vector that arises to ensure that the control requirements of the form of Eq. (5) are satisfied. The explicit equation of motion of the nominal system is given by the fundamental equation [13]:

$$
\mathbf{M} \ddot{q}=\boldsymbol{Q}+\mathbf{A}^{T}\left(\mathbf{A M}^{-1} \mathbf{A}^{T}\right)^{+}(\boldsymbol{b}-\mathbf{A} \boldsymbol{a})
$$

wherein the various quantities have been defined before, the superscript $T$ denotes the transpose of a vector or a matrix, and the superscript + denotes the Moore-Penrose inverse of a matrix. In the preceding equation, and in what follows, the arguments of the various quantities will be suppressed unless required for clarity. The control force that the uncontrolled system is subjected to, because of the presence of the control requirements of the form of Eq. (4), can be explicitly expressed as

$$
\boldsymbol{Q}^{c}(t):=\boldsymbol{Q}^{c}[\boldsymbol{q}(t), \dot{\boldsymbol{q}}(t), t]=\mathbf{A}^{T}\left(\mathbf{A} \mathbf{M}^{-1} \mathbf{A}^{T}\right)^{+}(\boldsymbol{b}-\mathbf{A} \boldsymbol{a})
$$

This control force minimizes the control cost $\boldsymbol{Q}^{c T} \mathbf{M}^{-1} \boldsymbol{Q}^{c}$ at each instant of time. The weighting matrix in the control cost has been chosen to be $\mathbf{M}^{-1}$, although other positive definite matrices can be easily chosen [15]. Equation (7) can be rewritten in the form 


$$
\ddot{\boldsymbol{q}}=\boldsymbol{a}+\mathbf{M}^{-1} \mathbf{A}^{T}\left(\mathbf{A M}^{-1} \mathbf{A}^{T}\right)^{+}(\boldsymbol{b}-\mathbf{A} \boldsymbol{a}):=\boldsymbol{a}+\mathbf{M}^{-1} \boldsymbol{Q}^{c}(t)
$$

a relation that shall be used later on.

The generalized control force given in Eq. (8) is predicated on the best assessment of the system, assuming that this assessment provides an accurate-enough deterministic model. Because in reallife situations uncertainties always exist, this control force $\boldsymbol{Q}^{c}(t)$ needs to be modified to compensate for these uncertainties.

As an example, consider uncertainties in the modeling of the masses and moments of inertia of the follower satellites. Such uncertainties in the mass could result from modeling errors in fuel consumption especially for long-duration missions. Errors in assessing the mass distribution may result in uncertainties in the determination of the moments of inertia. The latter could substantially affect the attitude control needed for, say, achieving the desired target pointing accuracy. These uncertainties affect the elements of the $n \times n$ matrix $\mathbf{M}$. Because the mass matrix $\mathbf{M}(\boldsymbol{q}, t)$, in general, is a function of the coordinate $\boldsymbol{q}$ and time $t$, variations in the mass and moments of inertia of the system cause changes in the coordinate $\boldsymbol{q}$ at each instance of time. Also, because the given force $\boldsymbol{Q}(\boldsymbol{q}, \dot{\boldsymbol{q}}, t)$ is also a function of the coordinate (and its derivative), uncertainty in the mass and moments of inertia will, in general, proliferate into the given force acting on the system.

Thus, to ensure that the follower satellites, whose models are not exactly known, track the orbital and attitude trajectory requirements of the nominal system, that is, they track the requirements of the bestestimate system, Eq. (6) has to be replaced with

$$
\mathbf{M}_{a}\left(\boldsymbol{q}_{c}, t\right) \ddot{\boldsymbol{q}}_{c}=\boldsymbol{Q}_{a}\left(\boldsymbol{q}_{c}, \dot{\boldsymbol{q}}_{c}, t\right)+\boldsymbol{Q}^{c}(t)+\boldsymbol{Q}^{u}(t)
$$

in which $\boldsymbol{q}_{c}$ is the generalized coordinate $n$-vector of the controlled actual system, and $\boldsymbol{Q}^{u}$ is the additional control force $n$-vector that compensates for the fact that the model is known only imprecisely, which shall be developed in closed form. The $n \times n$ matrix $\mathbf{M}_{a}:=$ $\mathbf{M}+\boldsymbol{\delta} \mathbf{M}>0$ is the actual mass matrix of the real-life system, which is a function of $\boldsymbol{q}_{c}$ and $t ; \boldsymbol{\delta} \mathbf{M}$ is the uncertainty in the mass matrix, which may include, among others, say, uncertainties in the masses and moments of inertia of the satellites; the actual given force vector is taken to be $\boldsymbol{Q}_{a}:=\boldsymbol{Q}+\boldsymbol{\delta} \boldsymbol{Q}$, in which the $n$-vector $\boldsymbol{Q}$ denotes the nominal given force, and $\boldsymbol{\delta} \boldsymbol{Q}$ denotes the $n$-vector of the changes in this given force that are caused by the presence of uncertainties in it, such as those caused by solar wind. The unconstrained acceleration of the actual uncertain system will be denoted by $\boldsymbol{a}_{a}:=\mathbf{M}_{a}^{-1} \boldsymbol{Q}_{a}$

Equation (10) is now referred to as the description of the controlled actual system, or controlled system, for short. Premultiplying both sides of Eq. (10) by $\mathbf{M}_{a}^{-1}$, the acceleration of the controlled system can be expressed as

$$
\ddot{\boldsymbol{q}}_{c}=\boldsymbol{a}_{a}+\mathbf{M}_{a}^{-1} \boldsymbol{Q}^{c}(t)+\mathbf{M}_{a}^{-1} \mathbf{M} \ddot{\boldsymbol{u}}_{c}
$$

Here, $\boldsymbol{a}_{a}:=\mathbf{M}_{a}^{-1} \boldsymbol{Q}_{a}$ and $\boldsymbol{Q}^{u}:=\mathbf{M} \ddot{\boldsymbol{u}}_{c}$, in which $\ddot{\boldsymbol{u}}_{c}$ is the additional generalized acceleration provided by the additional control forces $Q^{u}$ to compensate for uncertainties in the actual system, and is developed in Sec. V.

\section{Formation-Keeping Equations of Motion: The Controlled Nominal System}

It is assumed that there are $N$ follower satellites and that a leader satellite leads this $N$-satellite formation. The $i$ th follower satellite has a nominal mass $m^{(i)}$ and has a diagonal inertia matrix $\mathbf{J}^{(i)}$, in which the nominal moments of inertia along its body-fixed principal axes of inertia are placed.

It is also assumed that the position vector of the center of mass of the $i$ th follower satellite in the Hill frame [17] is given by $\left[\begin{array}{lll}x^{(i)} & y^{(i)} & z^{(i)}\end{array}\right]^{T}$, and its orientation is described by the quaternion $\boldsymbol{u}^{(i)}=\left[\begin{array}{llll}u_{0}^{(i)} & u_{1}^{(i)} & u_{2}^{(i)} & u_{3}^{(i)}\end{array}\right]^{T}$. Then, the generalized displacement 7 -vector is defined as

$$
\begin{gathered}
\boldsymbol{q}^{(i)}(t)=\left[\begin{array}{lllllll}
x^{(i)} & y^{(i)} & z^{(i)} & u_{0}^{(i)} & u_{1}^{(i)} & u_{2}^{(i)} & u_{3}^{(i)}
\end{array}\right]^{T}, \\
i=1,2, \ldots, N
\end{gathered}
$$

\section{A. Uncontrolled Orbital Motion}

The inertial orbital motion of the $i$ th follower satellite orbiting the spherical Earth is governed by the relation [20]

$$
\boldsymbol{a}_{\mathrm{ECI}}^{(i)}=\left[\begin{array}{c}
\ddot{X}^{(i)} \\
\ddot{Y}^{(i)} \\
\ddot{Z}^{(i)}
\end{array}\right]=-\frac{G M_{\oplus}}{\left(X^{(i)^{2}}+Y^{(i)^{2}}+Z^{(i)^{2}}\right)^{3 / 2}}\left[\begin{array}{c}
X^{(i)} \\
Y^{(i)} \\
Z^{(i)}
\end{array}\right]
$$

in which $\left[\begin{array}{lll}X^{(i)} & Y^{(i)} & Z^{(i)}\end{array}\right]^{T}$ is the position vector of the center of mass of the $i$ th follower satellite in the inertial frame or Earthcentered inertial (ECI) frame [20], $G$ is the universal gravitational constant, and $M_{\oplus}$ is the mass of Earth. The subscript ECI is used to stress that Eq. (13) is described in the ECI frame. In this paper, no perturbations and the Keplerian motion around a spherical Earth are assumed for simplicity, so as not to obscure the salient features of the proposed methodology.

As shown later, it is more convenient to use the Hill frame instead of the ECI frame in formation flying. The acceleration given by Eq. (13), when represented in the Hill frame, is given in [21] as

$$
\begin{gathered}
\boldsymbol{a}_{\mathrm{Hill}}^{(i)}=-\left[\begin{array}{c}
\ddot{r}_{L} \\
0 \\
0
\end{array}\right]-2 \mathbf{R} \dot{\mathbf{S}}\left[\begin{array}{c}
\dot{x}^{(i)}+\dot{r}_{L} \\
\dot{y}^{(i)} \\
\dot{z}^{(i)}
\end{array}\right]-\mathbf{R} \ddot{\mathbf{S}}\left[\begin{array}{c}
x^{(i)}+r_{L} \\
y^{(i)} \\
z^{(i)}
\end{array}\right] \\
-\frac{G M_{\oplus}}{\left[\left(x^{(i)}+r_{L}\right)^{2}+y^{(i)^{2}}+z^{(i)^{2}}\right]^{3 / 2}}\left[\begin{array}{c}
x^{(i)}+r_{L} \\
y^{(i)} \\
z^{(i)}
\end{array}\right]
\end{gathered}
$$

Here, the subscript Hill denotes that Eq. (14) is described in the Hill frame; $\left[\begin{array}{lll}x^{(i)} & y^{(i)} & z^{(i)}\end{array}\right]^{T}$ is the position vector of the center of mass of the $i$ th follower satellite in the Hill frame; $r_{L}$ is the distance from the center of Earth to the leader satellite, and the subscript $L$ denotes the leader satellite; and $\mathbf{R}$ is an orthogonal rotation matrix that maps the ECI frame to the Hill frame, that is

$$
\left[\begin{array}{c}
x^{(i)}+r_{L} \\
y^{(i)} \\
z^{(i)}
\end{array}\right]=\mathbf{R}\left[\begin{array}{c}
X^{(i)} \\
Y^{(i)} \\
Z^{(i)}
\end{array}\right]
$$

Each element of the matrix $\mathbf{R}$ is given in [21]. The matrix $\mathbf{S}$ in Eq. (14) is the active rotation matrix, which is the transpose of $\mathbf{R}$.

\section{B. Uncontrolled Rotational Motion}

As mentioned, the rotational dynamics of each follower satellite is described in terms of quaternions that obviate the troublesome problem of singularity. Recently, a new method to get the uncontrolled rotational equation of motion through the use of the fundamental equation was reported in [22], which is briefly derived as follows, and sets some of the notation that will be used. It follows the general approach for handling constrained motion problems: 1) the unconstrained system is first defined; 2) then, the constraints are defined; and 3) finally, these equations of the unconstrained motion and the constraints are used to get the constrained equations of motion directly using the fundamental equation of mechanics.

Lagrange's equation states that

$$
\frac{\mathrm{d}}{\mathrm{d} t}\left(\frac{\partial T^{(i)}}{\partial \dot{\boldsymbol{u}}^{(i)}}\right)-\frac{\partial T^{(i)}}{\partial \boldsymbol{u}^{(i)}}=\boldsymbol{\Gamma}_{u}^{(i)}\left(\boldsymbol{u}^{(i)}, \dot{\boldsymbol{u}}^{(i)}, t\right)
$$

in which $\boldsymbol{u}^{(i)}=\left[\begin{array}{llll}u_{0}^{(i)} & u_{1}^{(i)} & u_{2}^{(i)} & u_{3}^{(i)}\end{array}\right]^{T}$ is the quaternion 4-vector of the $i$ th follower satellite, $\boldsymbol{\Gamma}_{u}^{(i)}\left(\boldsymbol{u}^{(i)}, \dot{\boldsymbol{u}}^{(i)}, t\right)$ is the given generalized force 4-vector, and $T^{(i)}$ is the rotational kinetic energy of the $i$ th follower satellite, which is given by

$$
T^{(i)}=\frac{1}{2}\left\{\boldsymbol{\omega}^{(i)}\right\}^{T} \hat{\mathbf{J}}^{(i)}\left\{\boldsymbol{\omega}^{(i)}\right\}
$$


Here, the $4 \times 4$ augmented inertia matrix $\hat{\mathbf{J}}^{(i)}$ is defined as

$$
\hat{\mathbf{J}}^{(i)}:=\left[\begin{array}{c:c}
J_{0}^{(i)} & 0 \\
\hdashline 0 & \mathbf{J}^{(i)}
\end{array}\right]=\left[\begin{array}{c:ccc}
J_{0}^{(i)} & 0 & 0 & 0 \\
\hdashline 0 & J_{x}^{(i)} & 0 & 0 \\
0 & 0 & J_{y}^{(i)} & 0 \\
0 & 0 & 0 & J_{z}^{(i)}
\end{array}\right],
$$

in which $J_{0}^{(i)}$ is an arbitrary positive number, and $J_{x}^{(i)}, J_{y}^{(i)}, J_{z}^{(i)}$ are the moments of inertia along the principal axes of the $i$ th follower satellite. As shall be seen a little later, the addition of $J_{0}^{(i)}$ in the moment of inertia matrix permits the rotational kinetic energy when written in terms of quaternions to be positive definite. Also, the $4 \times 1$ augmented angular-velocity vector $\left\{\boldsymbol{\omega}^{(i)}\right\}$ is related with quaternions by

$$
\left\{\boldsymbol{\omega}^{(i)}\right\}=2 \mathbf{E}^{(i)} \dot{\boldsymbol{u}}^{(i)}, \quad i=1,2, \ldots, N
$$

in which $\left\{\boldsymbol{\omega}^{(i)}\right\}=\left[\begin{array}{llll}0 & \omega_{x}^{(i)} & \omega_{y}^{(i)} & \omega_{z}^{(i)}\end{array}\right]^{T}$, and the last three elements, described in the body frame, are the angular velocities about the ECI frame of reference, and the $4 \times 4$ quaternion matrix $\mathbf{E}^{(i)}$ is defined by

$$
\mathbf{E}^{(i)}:=\left[\begin{array}{l}
\boldsymbol{u}^{(i)^{\mathbf{T}}} \\
\overline{\mathbf{E}_{1}^{(i)}}
\end{array}\right]=\left[\begin{array}{cccc}
u_{0}^{(i)} & u_{1}^{(i)} & u_{2}^{(i)} & u_{3}^{(i)} \\
-\overline{-}-\overline{(i)} & u_{0}^{(i)} & -u_{3}^{(i)} & -u_{2}^{(i)} \\
-u_{2}^{(i)} & -u_{3}^{(i)} & u_{0}^{(i)} & u_{1}^{(i)} \\
-u_{3}^{(i)} & u_{2}^{(i)} & -u_{1}^{(i)} & u_{0}^{(i)}
\end{array}\right]
$$

Substituting Eq. (19) into Eq. (17) yields the kinetic energy in terms of quaternions

$$
T^{(i)}=2 \dot{\boldsymbol{u}}^{(i)^{T}} \mathbf{E}^{(i)^{T}} \hat{\mathbf{J}}^{(i)} \mathbf{E}^{(i)} \dot{\boldsymbol{u}}^{(i)}
$$

As mentioned before, this kinetic energy now is positive definite. Then, assuming that there is no applied torque (i.e., $\boldsymbol{\Gamma}_{u}^{(i)}=0$ ), Lagrange's equation is now applied under the assumption that the components of the quaternion 4-vector are all independent of each other to obtain

$$
4 \mathbf{E}^{(i)^{T}} \hat{\mathbf{J}}^{(i)} \mathbf{E}^{(i)} \ddot{\boldsymbol{u}}^{(i)}=-8 \dot{\mathbf{E}}^{(i)^{T}} \hat{\mathbf{J}}^{(i)} \mathbf{E}^{(i)} \dot{\boldsymbol{u}}^{(i)}-4 \mathbf{E}^{(i)^{T}} \hat{\mathbf{J}}^{(i)} \dot{\mathbf{E}}^{(i)} \dot{\boldsymbol{u}}^{(i)}
$$

This relation can be written in the form $\mathbf{M}_{u}^{(i)} \ddot{\boldsymbol{u}}^{(i)}=\boldsymbol{Q}_{u}^{(i)}$ by setting $\mathbf{M}_{u}^{(i)}:=4 \mathbf{E}^{(i)^{T}} \hat{\mathbf{J}}^{(i)} \mathbf{E}^{(i)}>0$, and $\boldsymbol{Q}_{u}^{(i)}$ to be the right-hand side of Eq. (22). It is noted that the mass matrix $\mathbf{M}_{u}^{(i)}=4 \mathbf{E}^{(i)^{T}} \hat{\mathbf{J}}^{(i)} \mathbf{E}^{(i)}$ is symmetric and positive definite, and so it has always its inverse.

It is important to stress that, up to now, it has been assumed that each component of the quaternion vector $\boldsymbol{u}^{(i)}$ is independent of the others. However, to represent a physical rotation of a rigid body, the quaternion $\boldsymbol{u}^{(i)}$ is required to have unit Euclidean norm, so that

$$
N_{u}\left(\boldsymbol{u}^{(i)}\right):=\left\|\boldsymbol{u}^{(i)}\right\|_{2}^{2}=u_{0}^{(i)^{2}}+u_{1}^{(i)^{2}}+u_{2}^{(i)^{2}}+u_{3}^{(i)^{2}}=1
$$

After differentiating twice, the following control requirement of the form of Eq. (5) is obtained as

$$
\left[\begin{array}{llll}
u_{0}^{(i)} & u_{1}^{(i)} & u_{2}^{(i)} & u_{3}^{(i)}
\end{array}\right]\left[\begin{array}{c}
\ddot{u}_{0}^{(i)} \\
\ddot{u}_{1}^{(i)} \\
\ddot{u}_{2}^{(i)} \\
\ddot{u}_{3}^{(i)}
\end{array}\right]=-\dot{u}_{0}^{(i)^{2}}-\dot{u}_{1}^{(i)^{2}}-\dot{u}_{2}^{(i)^{2}}-\dot{u}_{3}^{(i)^{2}}
$$

so that

$$
\begin{gathered}
\mathbf{A}_{u}^{(i)}=\left[\begin{array}{llll}
u_{0}^{(i)} & u_{1}^{(i)} & u_{2}^{(i)} & u_{3}^{(i)}
\end{array}\right] \\
\boldsymbol{b}_{u}^{(i)}=-\dot{u}_{0}^{(i)^{2}}-\dot{u}_{1}^{(i)^{2}}-\dot{u}_{2}^{(i)^{2}}-\dot{u}_{3}^{(i)^{2}}:=-N_{\dot{u}}\left(\dot{\boldsymbol{u}}^{(i)}\right)
\end{gathered}
$$

The resulting rotational equation of motion for the $i$ th follower satellite is thus given by Eq. (9) [22]:

$$
\ddot{\boldsymbol{u}}^{(i)}=-\frac{1}{2} \mathbf{E}_{1}^{(i)^{T}} \mathbf{J}^{(i)^{-1}}\left[\tilde{\boldsymbol{\omega}}^{(i)}\right] \mathbf{J}^{(i)} \boldsymbol{\omega}^{(i)}-N_{\dot{u}}\left(\dot{\boldsymbol{u}}^{(i)}\right) \boldsymbol{u}^{(i)}
$$

in which $\mathbf{E}_{1}^{(i)}, \mathbf{J}^{(i)}$, and $N_{\dot{u}}\left(\dot{\boldsymbol{u}}^{(i)}\right)$ are defined in Eqs. (20), (18), and $(\underline{25})$, respectively, and $\left[\tilde{\boldsymbol{\omega}}^{(i)}\right]$ is a skew-symmetric matrix defined by

$$
\left[\tilde{\boldsymbol{\omega}}^{(i)}\right]:=\left[\begin{array}{ccc}
0 & -\omega_{z}^{(i)} & \omega_{y}^{(i)} \\
\omega_{z}^{(i)} & 0 & -\omega_{x}^{(i)} \\
-\omega_{y}^{(i)} & \omega_{x}^{(i)} & 0
\end{array}\right]
$$

In Eq. (26), the 8-order system of differential equations using the quaternion and its derivative 8 -vector $(\boldsymbol{u}, \dot{\boldsymbol{u}})$ is used, although a 7 -order system could have been used, containing the angular velocity. Consistent with the Lagrangian approach used in developing these equations, the formulation resulting from the use of the generalized displacements defined in Eq. (12) employing the quaternion vectors $\boldsymbol{u}$ and $\dot{\boldsymbol{u}}$ has been retained. One can readily switch from one to the other using Eq. (19).

\section{Dynamics of Coupled Orbital and Rotational Motion of the Nominal System}

In this subsection, the attitude and orbital dynamics is combined. Upon defining the $7 \times 1$ generalized displacement vector $\boldsymbol{q}^{(i)}(t)$ as in Eq. (12), the following equation is obtained for the uncontrolled motion of each follower satellite from Eqs. (14) and (26):

$$
\begin{aligned}
\boldsymbol{a}^{(i)}(t) & =\left(\mathbf{M}^{(i)}\right)^{-1} \boldsymbol{Q}^{(i)} \\
& =\left[\begin{array}{c}
\boldsymbol{a}_{\text {Hill }}^{(i)} \\
--\mathbf{1}^{(} \mathbf{E}_{1}^{(i)^{\mathrm{T}}} \mathbf{J}^{(i)^{-1}}\left[\tilde{\boldsymbol{\omega}}^{(i)}\right] \mathbf{J}^{(i)} \boldsymbol{\omega}^{(i)}-N_{\dot{u}}\left(\dot{\boldsymbol{u}}^{(i)}\right) \boldsymbol{u}^{(i)}
\end{array}\right],
\end{aligned}
$$

in which the $7 \times 7$ mass matrix is

$$
\mathbf{M}^{(i)}=\left[\begin{array}{cc}
m^{(i)} \mathbf{I}_{3 \times 3} & \mathbf{0}_{3 \times 4} \\
\mathbf{0}_{4 \times 3} & \mathbf{M}_{u}^{(i)}
\end{array}\right]
$$

And the $4 \times 4$ matrix $\mathbf{M}_{u}^{(i)}:=4 \mathbf{E}^{(i)^{T}} \hat{\mathbf{J}}^{(i)} \mathbf{E}^{(i)}$ is previously defined, $\boldsymbol{a}_{\mathrm{Hill}}^{(i)}$ is a $3 \times 1$ vector on the right-hand side of Eq. (14), and $-1 / 2 \mathbf{E}_{1}^{(i)^{T}} \mathbf{J}^{(i)^{-1}}\left[\tilde{\boldsymbol{\omega}}^{(i)}\right] \boldsymbol{J}^{(i)} \boldsymbol{\omega}^{(i)}-N_{\dot{u}}\left(\dot{\boldsymbol{u}}^{(i)}\right) \boldsymbol{u}^{(i)}$ is a $4 \times 1$ vector on the right-hand side of Eq. (26).

Equation (28) describes the orbital and attitude dynamics of the $i$ th follower satellite when no control forces are applied to the system yet, so that it satisfies the desired attitude and orbital requirements.

When these trajectory/orientation requirements, which are of the form of Eq. (5), are imposed, the generalized control force required to follow them is explicitly obtained by Eq. (8), as shall be shown in the next subsection. In addition, one can relate the $4 \times 1$ generalized quaternion torque $\boldsymbol{\Gamma}_{u}^{(i)}$, which is determined by Eq. (16), to the $3 \times 1$ physically applied torque $\Gamma^{(i)}=\left[\begin{array}{lll}\Gamma_{x}^{(i)} & \Gamma_{y}^{(i)} & \Gamma_{z}^{(i)}\end{array}\right]^{T}$, about the body axis of the $i$ th follower satellite, through the relation [22]

$$
\left[\begin{array}{c}
0 \\
\boldsymbol{\Gamma}^{(i)}
\end{array}\right]=\frac{1}{2} \mathbf{E} \boldsymbol{\Gamma}_{u}^{(i)}, \quad i=1,2, \ldots, N
$$

\section{Determination of the Control Forces and Torques Using the Fundamental Equation}

In this subsection, an explicit form of the control force and torque is obtained via the fundamental equation, assuming no uncertainties in the masses and the moments of inertia of the follower satellites. These generalized forces are obtained based on the description of the nominal system. Also, it is assumed for brevity that there is only one follower satellite in the formation, and the leader satellite is in an 
unperturbed circular orbit with constant radius of $r_{L}$ around a spherical Earth. The following attitude and orbital requirements are considered: 1) the follower satellite's orbit should be on a circle with constant radius $\rho_{0}$ when projected onto the $y z$ plane of the Hill frame with the leader satellite located at the center of the circle, and this orbit is called the projected circular orbit (PCO) [23]; and 2) the follower satellite, more specifically, the $x$ axis of its body frame, points to the center of Earth at all times. 3) Besides these two requirements, the additional constraint is imposed that the quaternion 4-vector of the follower satellite should have unit norm, so that the quaternions represent rotations. Although it has already been contained in the derivation of Eq. (26), this constraint has been added for its importance. Unlike with the Lagrange multiplier method for enforcing constraints, applying the same constraints repeatedly has no ill effects in the approach used here. Then, these trajectory requirements are summarized as

$$
\begin{gathered}
2 x=z, \quad y=\rho_{0} \cos (\omega t), \quad z=\rho_{0} \sin (\omega t) \\
{\left[\tilde{\mathbf{x}}_{b}\right] \mathbf{P}\left[\begin{array}{l}
-X \\
-Y \\
-Z
\end{array}\right]=0}
\end{gathered}
$$

and

$$
N_{u}(\boldsymbol{u})=u_{0}^{2}+u_{1}^{2}+u_{2}^{2}+u_{3}^{2}=1
$$

in which $\left[\begin{array}{lll}x & y & z\end{array}\right]^{T}$ denotes the position in the Hill frame of the follower satellite, $\left[\begin{array}{lll}X & Y & Z\end{array}\right]^{T}$ is the position in the ECI frame, and $\omega$ in Eq. (31a) is a constant rotational frequency of the follower satellite about the leader satellite in the Hill frame. The position vector in the ECI frame in Eq. (31b) can be transformed to the one in the Hill frame, and vice versa, by using the relation:

$$
\left[\begin{array}{c}
X \\
Y \\
Z
\end{array}\right]=\mathbf{R}^{T}\left[\begin{array}{c}
x+r_{L} \\
y \\
z
\end{array}\right]=\left[\begin{array}{lll}
R_{11} & R_{21} & R_{31} \\
R_{12} & R_{22} & R_{32} \\
R_{13} & R_{23} & R_{33}
\end{array}\right]\left[\begin{array}{c}
x+r_{L} \\
y \\
z
\end{array}\right]
$$

in which the components of the transformation matrix $\mathbf{R}$ are given in [21], and $r_{L}$ is the constant distance between the leader satellite and the center of Earth. In Eq. (31b), $\left[\tilde{\mathbf{x}}_{b}\right]$ is the skew-symmetric matrix given by

$$
\left[\tilde{\mathbf{x}}_{b}\right]=\left[\begin{array}{ccc}
0 & 0 & 0 \\
0 & 0 & -1 \\
0 & 1 & 0
\end{array}\right]
$$

corresponding to the unit vector along the $x$ axis of the body frame $\hat{\boldsymbol{x}}_{b}=\left[\begin{array}{lll}1 & 0 & 0\end{array}\right]^{T}$, and $\mathbf{P}$ in Eq. (31b) is a transformation matrix that maps the ECI frame into the body frame of the follower satellite, which is of the form [22]

$$
\begin{aligned}
\mathbf{P} & =\left[\begin{array}{lll}
P_{11} & P_{12} & P_{13} \\
P_{21} & P_{22} & P_{23} \\
P_{31} & P_{32} & P_{33}
\end{array}\right] \\
& =\left[\begin{array}{ccc}
u_{0}^{2}+u_{1}^{2}-u_{2}^{2}-u_{3}^{2} & 2\left(u_{1} u_{2}+u_{0} u_{3}\right) & 2\left(u_{1} u_{3}-u_{0} u_{2}\right) \\
2\left(u_{1} u_{2}-u_{0} u_{3}\right) & u_{0}^{2}-u_{1}^{2}+u_{2}^{2}-u_{3}^{2} & 2\left(u_{0} u_{1}+u_{2} u_{3}\right) \\
2\left(u_{0} u_{2}+u_{1} u_{3}\right) & 2\left(u_{2} u_{3}-u_{0} u_{1}\right) & u_{0}^{2}-u_{1}^{2}-u_{2}^{2}+u_{3}^{2}
\end{array}\right]
\end{aligned}
$$

Equation $(\underline{31 \mathrm{~b}})$ originates from the fact that the desired pointing axis (i.e., $x$ axis of the body frame) is constrained to point along the vector connecting the follower satellite and the center of Earth in the ECI frame, $\left[\begin{array}{lll}-X & -Y & -Z\end{array}\right]^{T}$. The components of this vector, in turn, are transformed into the body frame by the transformation matrix $\mathbf{P}$, and the cross product of this transformed vector and the $x$ axis of the body frame is zero because they are parallel.

First, for the orbital requirements Eq. (31a), the following constraint equations are obtained by differentiating Eq. (31a) with respect to time twice:

$$
\mathbf{A}_{1} \ddot{\boldsymbol{q}}=\boldsymbol{b}_{1}
$$

in which the generalized displacement vector $\boldsymbol{q}$ is defined by Eq. (12) and

$$
\mathbf{A}_{1}=\left[\begin{array}{ccccccc}
2 & 0 & -1 & 0 & 0 & 0 & 0 \\
0 & 1 & 0 & 0 & 0 & 0 & 0 \\
0 & 0 & 1 & 0 & 0 & 0 & 0
\end{array}\right], \quad \boldsymbol{b}_{1}=\left[\begin{array}{c}
0 \\
-\omega^{2} \rho_{0} \cos (\omega t) \\
-\omega^{2} \rho_{0} \sin (\omega t)
\end{array}\right]
$$

Second, for the Earth-pointing attitude requirement, the following constraint equations are calculated by differentiating Eq. (31b) with respect to time twice:

$$
\mathbf{A}_{2} \ddot{\boldsymbol{q}}=\boldsymbol{b}_{2}
$$

in which

$$
\begin{aligned}
\mathbf{A}_{2} & =\left[\begin{array}{lllllll}
A_{2}^{(1,1)} & A_{2}^{(1,2)} & A_{2}^{(1,3)} & A_{2}^{(1,4)} & A_{2}^{(1,5)} & A_{2}^{(1,6)} & A_{2}^{(1,7)} \\
A_{2}^{(2,1)} & A_{2}^{(2,2)} & A_{2}^{(2,3)} & A_{2}^{(2,4)} & A_{2}^{(2,5)} & A_{2}^{(2,6)} & A_{2}^{(2,7)}
\end{array}\right], \\
\boldsymbol{b}_{2} & =\left[\begin{array}{l}
b_{2}^{(1)} \\
b_{2}^{(2)}
\end{array}\right]
\end{aligned}
$$

and

$$
\begin{aligned}
& A_{2}^{(1,1)}=P_{31} R_{11}+P_{32} R_{12}+P_{33} R_{13}, \quad A_{2}^{(1,2)}=P_{31} R_{21}+P_{32} R_{22}+P_{33} R_{23}, \quad A_{2}^{(1,3)}=P_{31} R_{31}+P_{32} R_{32}+P_{33} R_{33} \\
& A_{2}^{(1,4)}=2 u_{2}\left\{R_{11}\left(x+r_{L}\right)+R_{21} y+R_{31} z\right\}-2 u_{1}\left\{R_{12}\left(x+r_{L}\right)+R_{22} y+R_{32} z\right\}+2 u_{0}\left\{R_{13}\left(x+r_{L}\right)+R_{23} y+R_{33} z\right\} \\
& A_{2}^{(1,5)}=2 u_{3}\left\{R_{11}\left(x+r_{L}\right)+R_{21} y+R_{31} z\right\}-2 u_{0}\left\{R_{12}\left(x+r_{L}\right)+R_{22} y+R_{32} z\right\}-2 u_{1}\left\{R_{13}\left(x+r_{L}\right)+R_{23} y+R_{33} z\right\} \\
& A_{2}^{(1,6)}=2 u_{0}\left\{R_{11}\left(x+r_{L}\right)+R_{21} y+R_{31} z\right\}+2 u_{3}\left\{R_{12}\left(x+r_{L}\right)+R_{22} y+R_{32} z\right\}-2 u_{2}\left\{R_{13}\left(x+r_{L}\right)+R_{23} y+R_{33} z\right\} \\
& A_{2}^{(1,7)}=2 u_{1}\left\{R_{11}\left(x+r_{L}\right)+R_{21} y+R_{31} z\right\}+2 u_{2}\left\{R_{12}\left(x+r_{L}\right)+R_{22} y+R_{32} z\right\}+2 u_{3}\left\{R_{13}\left(x+r_{L}\right)+R_{23} y+R_{33} z\right\} \\
& A_{2}^{(2,1)}=-P_{21} R_{11}-P_{22} R_{12}-P_{23} R_{13}, \quad A_{2}^{(2,2)}=-P_{21} R_{21}-P_{22} R_{22}-P_{23} R_{23}, \quad A_{2}^{(2,3)}=-P_{21} R_{31}-P_{22} R_{32}-P_{23} R_{33} \\
& A_{2}^{(2,4)}=2 u_{3}\left\{R_{11}\left(x+r_{L}\right)+R_{21} y+R_{31} z\right\}-2 u_{0}\left\{R_{12}\left(x+r_{L}\right)+R_{22} y+R_{32} z\right\}-2 u_{1}\left\{R_{13}\left(x+r_{L}\right)+R_{23} y+R_{33} z\right\} \\
& A_{2}^{(2,5)}=-2 u_{2}\left\{R_{11}\left(x+r_{L}\right)+R_{21} y+R_{31} z\right\}+2 u_{1}\left\{R_{12}\left(x+r_{L}\right)+R_{22} y+R_{32} z\right\}-2 u_{0}\left\{R_{13}\left(x+r_{L}\right)+R_{23} y+R_{33} z\right\} \\
& A_{2}^{(2,6)}=-2 u_{1}\left\{R_{11}\left(x+r_{L}\right)+R_{21} y+R_{31} z\right\}-2 u_{2}\left\{R_{12}\left(x+r_{L}\right)+R_{22} y+R_{32} z\right\}-2 u_{3}\left\{R_{13}\left(x+r_{L}\right)+R_{23} y+R_{33} z\right\} \\
& A_{2}^{(2,7)}=2 u_{0}\left\{R_{11}\left(x+r_{L}\right)+R_{21} y+R_{31} z\right\}+2 u_{3}\left\{R_{12}\left(x+r_{L}\right)+R_{22} y+R_{32} z\right\}-2 u_{2}\left\{R_{13}\left(x+r_{L}\right)+R_{23} y+R_{33} z\right\}
\end{aligned}
$$




$$
\begin{aligned}
& b_{2}^{(1)}=-4\left(\dot{u}_{0} \dot{u}_{2}+\dot{u}_{1} \dot{u}_{3}\right)\left\{R_{11}\left(x+r_{L}\right)+R_{21} y+R_{31} z\right\} \\
& -4\left(\dot{u}_{2} \dot{u}_{3}-\dot{u}_{0} \dot{u}_{1}\right)\left\{R_{12}\left(x+r_{L}\right)+R_{22} y+R_{32} z\right\} \\
& -2\left(\dot{u}_{0}^{2}-\dot{u}_{1}^{2}-\dot{u}_{2}^{2}+\dot{u}_{3}^{2}\right)\left\{R_{13}\left(x+r_{L}\right)+R_{23} y+R_{33} z\right\} \\
& -4\left(\dot{u}_{0} u_{2}+u_{0} \dot{u}_{2}+\dot{u}_{1} u_{3}+u_{1} \dot{u}_{3}\right)\left\{\dot{R}_{11}\left(x+r_{L}\right)\right. \\
& \left.+\dot{R}_{21} y+R_{11} \dot{x}+R_{21} \dot{y}+R_{31} \dot{z}\right\} \\
& -4\left(\dot{u}_{2} u_{3}+u_{2} \dot{u}_{3}-\dot{u}_{0} u_{1}-u_{0} \dot{u}_{1}\right)\left\{\dot{R}_{12}\left(x+r_{L}\right)\right. \\
& \left.+\dot{R}_{22} y+R_{12} \dot{x}+R_{22} \dot{y}+R_{32} \dot{z}\right\} \\
& -4\left(u_{0} \dot{u}_{0}-u_{1} \dot{u}_{1}-u_{2} \dot{u}_{2}+u_{3} \dot{u}_{3}\right)\left\{\dot{R}_{13}\left(x+r_{L}\right)\right. \\
& \left.+\dot{R}_{23} y+R_{13} \dot{x}+R_{23} \dot{y}+R_{33} \dot{z}\right\} \\
& -P_{31}\left\{\ddot{R}_{11}\left(x+r_{L}\right)+\ddot{R}_{21} y+2 \dot{R}_{11} \dot{x}+2 \dot{R}_{21} \dot{y}\right\} \\
& -P_{32}\left\{\ddot{R}_{12}\left(x+r_{L}\right)+\ddot{R}_{22} y+2 \dot{R}_{12} \dot{x}+2 \dot{R}_{22} \dot{y}\right\} \\
& -P_{33}\left\{\ddot{R}_{13}\left(x+r_{L}\right)+\ddot{R}_{23} y+2 \dot{R}_{13} \dot{x}+2 \dot{R}_{23} \dot{y}\right\} \\
& b_{2}^{(2)}=4\left(\dot{u}_{1} \dot{u}_{2}-\dot{u}_{0} \dot{u}_{3}\right)\left\{R_{11}\left(x+r_{L}\right)+R_{21} y+R_{31} z\right\} \\
& +2\left(\dot{u}_{0}^{2}-\dot{u}_{1}^{2}+\dot{u}_{2}^{2}-\dot{u}_{3}^{2}\right)\left\{R_{12}\left(x+r_{L}\right)+R_{22} y+R_{32} z\right\} \\
& +4\left(\dot{u}_{0} \dot{u}_{1}+\dot{u}_{2} \dot{u}_{3}\right)\left\{R_{13}\left(x+r_{L}\right)+R_{23} y+R_{33} z\right\} \\
& +4\left(\dot{u}_{1} u_{2}+u_{1} \dot{u}_{2}-\dot{u}_{0} u_{3}-u_{0} \dot{u}_{3}\right)\left\{\dot{R}_{11}\left(x+r_{L}\right)\right. \\
& \left.+\dot{R}_{21} y+R_{11} \dot{x}+R_{21} \dot{y}+R_{31} \dot{z}\right\} \\
& +4\left(u_{0} \dot{u}_{0}-u_{1} \dot{u}_{1}+u_{2} \dot{u}_{2}-u_{3} \dot{u}_{3}\right)\left\{\dot{R}_{12}\left(x+r_{L}\right)\right. \\
& \left.+\dot{R}_{22} y+R_{12} \dot{x}+R_{22} \dot{y}+R_{32} \dot{z}\right\} \\
& +4\left(\dot{u}_{0} u_{1}+u_{0} \dot{u}_{1}+\dot{u}_{2} u_{3}+u_{2} \dot{u}_{3}\right)\left\{\dot{R}_{13}\left(x+r_{L}\right)\right. \\
& \left.+\dot{R}_{23} y+R_{13} \dot{x}+R_{23} \dot{y}+R_{33} \dot{z}\right\} \\
& +P_{21}\left\{\ddot{R}_{11}\left(x+r_{L}\right)+\ddot{R}_{21} y+2 \dot{R}_{11} \dot{x}+2 \dot{R}_{21} \dot{y}\right\} \\
& +P_{22}\left\{\ddot{R}_{12}\left(x+r_{L}\right)+\ddot{R}_{22} y+2 \dot{R}_{12} \dot{x}+2 \dot{R}_{22} \dot{y}\right\} \\
& +P_{23}\left\{\ddot{R}_{13}\left(x+r_{L}\right)+\ddot{R}_{23} y+2 \dot{R}_{13} \dot{x}+2 \dot{R}_{23} \dot{y}\right\}
\end{aligned}
$$

Here, the fact that the elements $R_{31}, R_{32}$, and $R_{33}$ are constants is used because the leader satellite is in an unperturbed circular orbit [17].

Finally, the following constraint equations for the unit-norm quaternion constraint Eq. (31c) are obtained:

$$
\mathbf{A}_{3} \ddot{q}=\boldsymbol{b}_{3}
$$

in which

$$
\begin{aligned}
\mathbf{A}_{3} & =\left[\begin{array}{lllllll}
0 & 0 & 0 & u_{0} & u_{1} & u_{2} & u_{3}
\end{array}\right], \\
\boldsymbol{b}_{3} & =-\dot{u}_{0}^{2}-\dot{u}_{1}^{2}-\dot{u}_{2}^{2}-\dot{u}_{3}^{2}
\end{aligned}
$$

In conclusion, the following constraint equations are obtained, which are of the form of Eq. (5):

$$
\left[\begin{array}{l}
\mathbf{A}_{1} \\
\mathbf{A}_{2} \\
\mathbf{A}_{3}
\end{array}\right] \ddot{\boldsymbol{q}}=\left[\begin{array}{l}
\boldsymbol{b}_{1} \\
\boldsymbol{b}_{2} \\
\boldsymbol{b}_{3}
\end{array}\right]
$$

in which each element is given in Eqs. (36), (38), (39), and (41). Then, the control force and torque for the nominal system to satisfy the given orbital and attitude requirements are explicitly determined by Eq. ().

\section{Uncertainties in the Dynamics of Satellite Systems}

Real-life multisatellite systems usually have uncertainties, which, in general, arise due to the lack of precise knowledge of the physical system and/or of the given forces applied to the system. With the conceptualization of the nominal system given in the previous sections, these uncertainties are now assumed to be encapsulated in the elements of the $n \times n$ matrix $\mathbf{M}$ and the $n$-vector $\boldsymbol{Q}$ [see Eq. (1)] These uncertainties cause an error in satisfying the desired control requirements of the form of Eq. (4) and result in a difference between the trajectories of the real-life uncertain system and the nominal system.

Let us start by defining the tracking-error signal as

$$
\boldsymbol{e}(t)=\boldsymbol{q}_{c}(t)-\boldsymbol{q}(t)
$$

Differentiating Eq. (43) twice with respect to time, one can get

$$
\ddot{\boldsymbol{e}}=\ddot{\boldsymbol{q}}_{c}-\ddot{\boldsymbol{q}}
$$

which, upon use of Eqs. (9) and (11), yields

$$
\begin{aligned}
\ddot{\boldsymbol{e}} & =\left[\boldsymbol{a}_{a}\left(\boldsymbol{q}_{c}, \dot{\boldsymbol{q}}_{c}, t\right)-\boldsymbol{a}(\boldsymbol{q}, \dot{\boldsymbol{q}}, t)\right]+\left[\mathbf{M}_{a}^{-1}\left(\boldsymbol{q}_{c}, t\right)-\mathbf{M}^{-1}(\boldsymbol{q}, t)\right] \boldsymbol{Q}^{c}(t) \\
& +\mathbf{M}_{a}^{-1} \mathbf{M} \ddot{\boldsymbol{u}}_{c} \\
& :=\delta \ddot{\boldsymbol{q}}+\mathbf{M}_{a}^{-1} \mathbf{M} \ddot{\boldsymbol{u}}_{c}=\delta \ddot{\boldsymbol{q}}+\left[\mathbf{I}-\left(\mathbf{I}-\mathbf{M}_{a}^{-1} \mathbf{M}\right)\right] \ddot{\boldsymbol{u}}_{c}:=\delta \ddot{\boldsymbol{q}}+\ddot{\boldsymbol{u}}_{c}-\overline{\mathbf{M}} \ddot{\boldsymbol{u}}_{c}
\end{aligned}
$$

In the preceding equation,

$$
\begin{aligned}
\overline{\mathbf{M}} & =\mathbf{I}-\mathbf{M}_{a}^{-1}\left(\boldsymbol{q}_{c}, t\right) \mathbf{M}(\boldsymbol{q}, t)=\mathbf{I}-\left[\mathbf{M}\left(\boldsymbol{q}_{c}, t\right)+\boldsymbol{\delta} \mathbf{M}\left(\boldsymbol{q}_{c}, t\right)\right]^{-1} \mathbf{M}(\boldsymbol{q}, t) \\
& =\mathbf{I}-\left[\mathbf{M}^{-1}(\boldsymbol{q}, t) \mathbf{M}\left(\boldsymbol{q}_{c}, t\right)+\mathbf{M}^{-1}(\boldsymbol{q}, t) \boldsymbol{\delta} \mathbf{M}\left(\boldsymbol{q}_{c}, t\right)\right]^{-1}
\end{aligned}
$$

and the acceleration $\delta \ddot{\boldsymbol{q}}$ is given by

$$
\begin{aligned}
& \delta \ddot{\boldsymbol{q}}\left(\boldsymbol{q}, \dot{\boldsymbol{q}}, \boldsymbol{q}_{c}, \dot{\boldsymbol{q}}_{c}, t\right)=\left[\boldsymbol{a}_{a}\left(\boldsymbol{q}_{c}, \dot{\boldsymbol{q}}_{c}, t\right)-\boldsymbol{a}(\boldsymbol{q}, \dot{\boldsymbol{q}}, t)\right] \\
& +\left[\mathbf{M}_{a}^{-1}\left(\boldsymbol{q}_{c}, t\right)-\mathbf{M}^{-1}(\boldsymbol{q}, t)\right] \boldsymbol{Q}^{c}(t)
\end{aligned}
$$

in which $\boldsymbol{a}_{a}:=\mathbf{M}_{a}^{-1} \boldsymbol{Q}_{a}$, with $\mathbf{M}_{a}:=\mathbf{M}\left(\boldsymbol{q}_{c}, t\right)+\boldsymbol{\delta} \mathbf{M}\left(\boldsymbol{q}_{c}, t\right)$ and $\boldsymbol{Q}_{a}:=\boldsymbol{Q}\left(\boldsymbol{q}_{c}, \dot{\boldsymbol{q}}_{c}, t\right)+\boldsymbol{\delta} \boldsymbol{Q}\left(\boldsymbol{q}_{c}, \dot{\boldsymbol{q}}_{c}, t\right)$.

The aim in this section is to find a suitable bound on $\delta \ddot{\boldsymbol{q}}$, which shall be used in the following section to develop a set of continuous additive controllers to compensate for the uncertainties involved in the knowledge of the actual satellite system.

Using Taylor's expansion, Eq. (47) can be expanded as

$\delta \ddot{\boldsymbol{q}}\left(\boldsymbol{q}, \dot{\boldsymbol{q}}, \boldsymbol{q}_{c}, \dot{\boldsymbol{q}}_{c}, t\right)=\mathbf{M}_{a}^{-1}(\boldsymbol{q}, t) \boldsymbol{Q}_{a}(\boldsymbol{q}, \dot{\boldsymbol{q}}, t)-\mathbf{M}^{-1}(\boldsymbol{q}, t) \boldsymbol{Q}(\boldsymbol{q}, \dot{\boldsymbol{q}}, t)$

$+\mathbf{M}_{a}^{-1}(\boldsymbol{q}, t)\left[\left.\sum_{j=1}^{n} \frac{\partial \boldsymbol{Q}_{a, i}}{\partial \boldsymbol{q}_{c j}}\right|_{\boldsymbol{q}, \dot{\boldsymbol{q}}, t}\left(\boldsymbol{q}_{c j}-\boldsymbol{q}_{j}\right)+\left.\sum_{j=1}^{n} \frac{\partial \boldsymbol{Q}_{a, i}}{\partial \dot{\boldsymbol{q}}_{c j}}\right|_{\boldsymbol{q}, \dot{\boldsymbol{q}}, t}\left(\dot{\boldsymbol{q}}_{c j}-\dot{\boldsymbol{q}}_{j}\right)\right]$

$+\left[\left.\sum_{j=1}^{n} \frac{\partial \mathbf{M}_{a, i k}^{-1}}{\partial \boldsymbol{q}_{c j}}\right|_{\boldsymbol{q}, t}\left(\boldsymbol{q}_{c j}-\boldsymbol{q}_{j}\right)\right]$

$\times\left[\boldsymbol{Q}_{a}(\boldsymbol{q}, \dot{\boldsymbol{q}}, t)+\left.\sum_{j=1}^{n} \frac{\partial \boldsymbol{Q}_{a, i}}{\partial \boldsymbol{q}_{c j}}\right|_{\boldsymbol{q}, \dot{\boldsymbol{q}}, t}\left(\boldsymbol{q}_{c j}-\boldsymbol{q}_{j}\right)+\left.\sum_{j=1}^{n} \frac{\partial \boldsymbol{Q}_{a, i}}{\partial \dot{\boldsymbol{q}}_{c j}}\right|_{\boldsymbol{q}, \dot{\boldsymbol{q}}, t}\left(\dot{\boldsymbol{q}}_{c j}-\dot{\boldsymbol{q}}_{j}\right)\right]$

$+\left\{\mathbf{M}_{a}^{-1}(\boldsymbol{q}, t)+\left[\left.\sum_{j=1}^{n} \frac{\partial \mathbf{M}_{a, i k}^{-1}}{\partial \boldsymbol{q}_{c j}}\right|_{\boldsymbol{q}, t}\left(\boldsymbol{q}_{c j}-\boldsymbol{q}_{j}\right)\right]-\mathbf{M}^{-1}(\boldsymbol{q}, t)\right\} \boldsymbol{Q}^{c}(t)$

+ H.O.T, for $i=1, \ldots, n$ and $k=1, \ldots, n$

in which H.O.T. denotes the higher-order terms of $\left(\boldsymbol{q}_{c}-\boldsymbol{q}\right)$ and $\left(\dot{\boldsymbol{q}}_{c}-\dot{\boldsymbol{q}}\right)$.

It is noted that, in Eq. (48), $\boldsymbol{Q}_{a, i}, \boldsymbol{q}_{c j}$, and $\boldsymbol{q}_{j}$ denote the corresponding $i$ th and $j$ th components of the $n$-vectors $\boldsymbol{Q}_{a}, \boldsymbol{q}_{c}$, and $\boldsymbol{q}$, respectively. Also, $\mathbf{M}_{a, i k}^{-1}$ represents the $(i, k)$ element of the $n \times n$ matrix $\mathbf{M}_{a}^{-1}$.

The aim is to develop a controller $\ddot{\boldsymbol{u}}_{c}$, such that the motion of the controlled actual system closely tracks the motion of the nominal system, and thereby satisfies the control requirements of the form of Eq. (4). It is assumed for the moment that the compensating control acceleration $\ddot{\boldsymbol{u}}_{c}$ is capable of this and causes the trajectory of the controlled actual system $\left(\boldsymbol{q}_{c}, \dot{\boldsymbol{q}}_{c}\right)$ to sufficiently approximate that of the nominal system, so that $\left(\boldsymbol{q}_{c}, \dot{\boldsymbol{q}}_{c}\right) \approx(\boldsymbol{q}, \dot{\boldsymbol{q}})$. Under this assumption, 
one can take the lowest-order terms in Eq. (48), and approximate $\delta \ddot{\boldsymbol{q}}$ as

$$
\begin{aligned}
& \delta \ddot{\boldsymbol{q}}(\boldsymbol{q}, \dot{\boldsymbol{q}}, t)=\left[\mathbf{M}_{a}^{-1}(\boldsymbol{q}, t) \boldsymbol{Q}_{a}(\boldsymbol{q}, \dot{\boldsymbol{q}}, t)-\mathbf{M}^{-1}(\boldsymbol{q}, t) \boldsymbol{Q}(\boldsymbol{q}, \dot{\boldsymbol{q}}, t)\right] \\
& +\left[\mathbf{M}_{a}^{-1}(\boldsymbol{q}, t)-\mathbf{M}^{-1}(\boldsymbol{q}, t)\right] \boldsymbol{Q}^{c}(t)
\end{aligned}
$$

Similarly, one can take the lowest-order terms in Eq. (46) and assume that $\left\|\mathbf{M}^{-1} \boldsymbol{\delta} \mathbf{M}\right\| \ll 1$. Thus, by Taylor's expansion, $\overline{\mathbf{M}}$ in Eq. (46) can be approximated as

$$
\overline{\mathbf{M}} \approx \mathbf{I}-\left[\mathbf{I}+\mathbf{M}^{-1}(\boldsymbol{q}, t) \boldsymbol{\delta} \mathbf{M}(\boldsymbol{q}, t)\right]^{-1} \approx \mathbf{M}^{-1} \boldsymbol{\delta} \mathbf{M}
$$

Since [24]

$$
\begin{aligned}
& \mathbf{M}_{a}^{-1}(\boldsymbol{q}, t)=[\mathbf{M}(\boldsymbol{q}, t)+\boldsymbol{\delta} \mathbf{M}(\boldsymbol{q}, t)]^{-1} \\
& =\mathbf{M}^{-1}-\mathbf{M}^{-1}\left(\mathbf{I}+\boldsymbol{\delta} \mathbf{M} \mathbf{M}^{-1}\right)^{-1} \boldsymbol{\delta} \mathbf{M M}^{-1}
\end{aligned}
$$

expanding Eq. (49) and utilizing Eq. (1), one can obtain

$$
\delta \ddot{\boldsymbol{q}} \approx-(\mathbf{M}+\boldsymbol{\delta} \mathbf{M})^{-1} \boldsymbol{\delta} \mathbf{M M}^{-1}\left(\boldsymbol{Q}+\boldsymbol{Q}^{c}\right)+(\mathbf{M}+\boldsymbol{\delta} \mathbf{M})^{-1} \boldsymbol{\delta} \boldsymbol{Q}
$$

which includes the combined effect of the uncertainties $\boldsymbol{\delta} \mathbf{M}$ and $\boldsymbol{\delta} \boldsymbol{Q}$. By taking the norm of Eq. (2), one can obtain an estimate of the bound, $\gamma(t)$, on $\|\boldsymbol{\delta} \ddot{\boldsymbol{q}}\|$ as

$$
\begin{aligned}
& \|\delta \ddot{\boldsymbol{q}}(t)\| \approx \|-(\mathbf{M}+\boldsymbol{\delta} \mathbf{M})^{-1} \boldsymbol{\delta} \mathbf{M M}^{-1}\left(\boldsymbol{Q}+\boldsymbol{Q}^{c}\right) \\
& +(\mathbf{M}+\boldsymbol{\delta} \mathbf{M})^{-1} \boldsymbol{\delta} \boldsymbol{Q} \| \leq \gamma(t)
\end{aligned}
$$

in which $\gamma(t)$ is a positive function of time.

While the framework developed here is sufficient for considering any one (or all simultaneously) of the uncertainties in the mass matrix and in the given generalized force vector of the dynamics of the actual satellite systems, later on in this paper, for illustrative purposes, uncertainties in the mass and moments of inertia of the follower satellite are considered.

\section{Generalized Sliding-Surface Controller}

Having obtained an estimate of the bound $\gamma$, the aim in this section is to develop a compensating controller that can guarantee tracking (to within desired error bounds) of the nominal system's trajectory in the presence of uncertainties in the actual satellite system. To do this, a generalization of the concept of a sliding surface [25-28] is used. The formulation permits the use of a large class of control laws that can be adapted to the practical limitations of the specific compensating (continuous) controller being used, and the extent to which it is desired to compensate for the uncertainties.

Noting Eq. (45), the tracking-error signal in acceleration can be expressed as

$$
\ddot{\boldsymbol{e}}=\boldsymbol{\delta} \ddot{\boldsymbol{q}}+\mathbf{M}_{a}^{-1} \mathbf{M} \ddot{\boldsymbol{u}}_{c}:=\boldsymbol{\delta} \ddot{\boldsymbol{q}}+\ddot{\boldsymbol{u}}_{c}-\overline{\mathbf{M}} \ddot{\boldsymbol{u}}_{c}
$$

in which $\overline{\mathbf{M}} \approx \mathbf{I}-\left(\mathbf{I}+\mathbf{M}^{-1} \boldsymbol{\delta} \mathbf{M}\right)^{-1}$ has been used in the last equality. Let us now define a sliding surface:

$$
\boldsymbol{s}(t)=k e(t)+\dot{e}(t)
$$

in which $k>0$ is an arbitrary small positive number, and $s$ is an $n$ vector. The aim is to maneuver the system to the sliding surface $s \in \Omega_{\varepsilon}$, whereupon by Eq. (55), ideally speaking, when the size of the surface $\Omega_{\varepsilon}$ is zero, the relation $\dot{\boldsymbol{e}}=-k \boldsymbol{e}$ is obtained, whose solution $\boldsymbol{e}(t)=\boldsymbol{e}_{0} \exp (-k t)$ shows that the tracking error $\boldsymbol{e}(t)$ exponentially reduces to zero along this lower-dimensional surface in phase space.

Differentiating Eq. (55) with respect to time and using Eq. (54) yield

$$
\dot{\boldsymbol{s}}=k \dot{\boldsymbol{e}}+\ddot{\boldsymbol{e}}=k \dot{\boldsymbol{e}}+\delta \ddot{\boldsymbol{q}}+\ddot{\boldsymbol{u}}_{c}-\overline{\mathbf{M}} \ddot{\boldsymbol{u}}_{c}
$$

Since $\left(\dot{\boldsymbol{q}}_{c}-\dot{\boldsymbol{q}}\right)$ can be measured, to cancel the known term $k \dot{\boldsymbol{e}}=$ $k\left(\dot{\boldsymbol{q}}_{c}-\dot{\boldsymbol{q}}\right)$ in Eq. $(\underline{56})$, the controller $\ddot{\boldsymbol{u}}_{c}$ is chosen to be of the form

$$
\ddot{\boldsymbol{u}}_{c}=-k \dot{\boldsymbol{e}}(t)+\boldsymbol{v}(t)
$$

so that

$$
\dot{\boldsymbol{s}}=\boldsymbol{v}+\boldsymbol{\delta} \ddot{\boldsymbol{q}}-\overline{\mathbf{M}}[-k \dot{\boldsymbol{e}}(t)+\boldsymbol{v}(t)]
$$

It is noted that $\|\delta \ddot{\boldsymbol{q}}\| \leq \gamma(t)$. Here, the bound $\gamma(t)$ is used, which is related to the uncertainties involved in the real-life satellite system, and is obtained from Eq. (53). In what follows, $\|\cdot\|$ shall be denoted to mean the infinity norm.

Now, it will be shown that the system can indeed be maneuvered to the sliding surface $s \in \Omega_{\varepsilon}$ when $\Omega_{\varepsilon}$ is defined as an appropriately small surface around $s=0$, whose exact description will be shortly discussed.

Consider a function $\beta(t)$, such that

$$
\beta(t) \geq \frac{n\left[\gamma(t)+\beta_{0}\right]}{\alpha_{0}}>0
$$

in which

$$
\beta_{0}>k\|\overline{\mathbf{M}}(t)\|\|\dot{\boldsymbol{e}}(t)\|, 0<\alpha_{0}<1-n\|\overline{\mathbf{M}}(t)\|
$$

are any arbitrary positive constants over the time duration over which the control is applied.

Let a control $n$-vector $v(t)$ be defined, so that

$$
v(t):=-\beta(t) f(s)
$$

The $i$ th component, $f_{i}(s)$, of the $n$-vector $f(s)$ is defined as

$$
f_{i}(s)=g\left(s_{i} / \varepsilon\right), \quad i=1, \ldots, n
$$

in which $s_{i}$ is the $i$ th component of the $n$-vector $s ; \varepsilon$ is defined as any (small) positive number; and the function $g\left(s_{i} / \varepsilon\right)$ is any arbitrary monotonically increasing, continuous, odd function of $s_{i}$ on the interval $(-\infty,+\infty)$ that satisfies

$$
\|\boldsymbol{f}(\boldsymbol{s})\|=\|g(\boldsymbol{s} / \varepsilon)\| \geq \frac{\gamma(t)+k\|\overline{\mathbf{M}}(t)\|\|\dot{\boldsymbol{e}}(t)\|}{\gamma(t)+\beta_{0}},
$$

if $s$ is outside the surface $\Omega_{\varepsilon}(t)$

in which $\Omega_{\varepsilon}(t)$ is defined as the surface of the $n$-dimensional cube around the point $s=0$, each of whose sides has a computable length (as shown below). It is noted that the right-hand side of Eq. (63) is always less than unity since $\beta_{0}>k\|\overline{\mathbf{M}}(t)\|\|\dot{\boldsymbol{e}}(t)\|$, and hence, Eq. (63) will always be satisfied when $\|f(s)\| \geq 1$.

Result: The control law

$$
\ddot{\boldsymbol{u}}_{c}=-k \dot{\boldsymbol{e}}(t)+\boldsymbol{v}(t)=-[k \dot{\boldsymbol{e}}(t)+\beta(t) \boldsymbol{f}(\boldsymbol{s})]
$$

with $k>0$ and $v(t)$ defined in Eqs. (61-63) will cause $s(t) \rightarrow \Omega_{\varepsilon}$.

Proof: Consider the Lyapunov function:

$$
V=\frac{1}{2} \boldsymbol{s}^{T} \boldsymbol{s}
$$

Differentiating Eq. (65) once with respect to time yields

$$
\dot{V}=s^{T} \dot{s}
$$

Substituting Eq. (하) in Eq. (66), one can have

$$
\dot{V}=\boldsymbol{s}^{T}(t) \boldsymbol{v}(t)+\boldsymbol{s}^{T}(t) \delta \ddot{\boldsymbol{q}}(t)+k \boldsymbol{s}^{T}(t) \overline{\mathbf{M}}(t) \dot{\boldsymbol{e}}(t)-\boldsymbol{s}^{T}(t) \overline{\mathbf{M}}(t) \boldsymbol{v}(t)
$$

Then, upon using Eq. (61) in Eq. (67), the following is obtained:

$$
\dot{V}=-\beta \boldsymbol{s}^{T} \boldsymbol{f}(\boldsymbol{s})+\boldsymbol{s}^{T} \delta \ddot{\boldsymbol{q}}+k \boldsymbol{s}^{T} \overline{\mathbf{M}} \dot{\boldsymbol{e}}+\beta \boldsymbol{s}^{T} \overline{\mathbf{M}} \boldsymbol{f}(\boldsymbol{s})
$$


so that

$$
\dot{V} \leq-\beta \boldsymbol{s}^{T} \boldsymbol{f}(\boldsymbol{s})+\left\|\boldsymbol{s}^{T}\right\|\|\boldsymbol{\delta} \ddot{\boldsymbol{q}}\|+k\left\|\boldsymbol{s}^{T}\right\|\|\overline{\mathbf{M}}\|\|\dot{\boldsymbol{e}}\|+\beta\left\|\boldsymbol{s}^{T}\right\|\|\overline{\mathbf{M}}\|\|\boldsymbol{f}(\boldsymbol{s})\|
$$

Then, using relation $\|\boldsymbol{\delta} \ddot{\boldsymbol{q}}\| \leq \gamma(t)$ and noting $\left\|\boldsymbol{s}^{T}\right\| \leq n\|\boldsymbol{s}\|$, one can get

$$
\dot{V} \leq-\beta \boldsymbol{s}^{T} \boldsymbol{f}(\boldsymbol{s})+n\|\boldsymbol{s}\| \gamma(t)+n k\|\boldsymbol{s}\|\|\overline{\mathbf{M}}\|\|\dot{\boldsymbol{e}}\|+n \beta\|\boldsymbol{s}\|\|\overline{\mathbf{M}}\|\|\boldsymbol{f}(\boldsymbol{s})\|
$$

Because $f(s)$ is an odd monotonically function of $s$, and $s$ and $f(s)$ have the same sign, the following is satisfied:

$$
s^{T} f(s) \geq\|s\|\|f(s)\|
$$

Using Eq. (71), thus Eq. (70) becomes

$$
\begin{aligned}
\dot{V} & \leq-\|\boldsymbol{s}\|(\beta\|\boldsymbol{f}(\boldsymbol{s})\|-n \beta\|\overline{\mathbf{M}}\|\|\boldsymbol{f}(\boldsymbol{s})\|-n \gamma(t)-n k\|\overline{\mathbf{M}}\|\|\dot{\boldsymbol{e}}\|) \\
& =-\|\boldsymbol{s}\|[\beta(1-n\|\overline{\mathbf{M}}\|)\|\boldsymbol{f}(\boldsymbol{s})\|-n \gamma(t)-n k\|\overline{\mathbf{M}}\|\|\dot{\boldsymbol{e}}\|] \\
& =-n\|\boldsymbol{s}\|\left[\frac{\beta}{n}(1-n\|\overline{\mathbf{M}}\|)\|\boldsymbol{f}(\boldsymbol{s})\|-\gamma(t)-k\|\overline{\mathbf{M}}\|\|\dot{\boldsymbol{e}}\|\right]
\end{aligned}
$$

Since $\beta \geq n\left[\gamma(t)+\beta_{0}\right] / \alpha_{0}$, in which $0<\alpha_{0}<(1-n\|\overline{\mathbf{M}}\|)$

$$
\dot{V} \leq-n\|\boldsymbol{s}\|\left[\left(\gamma(t)+\beta_{0}\right)\|\boldsymbol{f}(\boldsymbol{s})\|-\gamma(t)-k\|\overline{\mathbf{M}}\|\|\dot{\boldsymbol{e}}\|\right]
$$

Because by Eq. $(63),\left(\gamma(t)+\beta_{0}\right)\|f(s)\|-\gamma(t)-k\|\overline{\mathbf{M}}(t)\|\|\dot{\boldsymbol{e}}(t)\|$ : $=\Delta(t) \geq 0$ outside the surface $\Omega_{\varepsilon}(t)$, it follows that

$$
\dot{V} \leq-n\|s\| \Delta(t), \quad \text { outside the surface } \Omega_{\varepsilon}(t)
$$

so that the derivative $\dot{V}$ is negative, and convergence to the closed set interior to the region enclosed by the surface $\Omega_{\varepsilon}(t)$ is guaranteed. $\square$

Note that, for Eq. (74) to be satisfied, relation Eq. (63) is required, namely

$$
\|\boldsymbol{f}(\boldsymbol{s})\|=\|g(\boldsymbol{s} / \varepsilon)\| \geq \frac{\gamma(t)+k\|\overline{\mathbf{M}}(t)\|\|\dot{\boldsymbol{e}}(t)\|}{\gamma(t)+\beta_{0}}:=\Xi(t)
$$

in which, as noted before, $\Xi(t)<1$. Equation ( $\underline{75})$ then yields

$$
\|\boldsymbol{s}\| \geq \varepsilon\left\|g^{-1}[\Xi(t)]\right\|
$$

In the region in which $\|s\|$ satisfies Eq. (76), the Lyapunov derivative $\dot{V}$ is negative. This proves that the controller described in Eq. (64) will cause $s(t)$ to decrease until it reaches the boundary $s \in \Omega_{\varepsilon}(t)$. Further, since $\Xi(t)<1$, and the function $g(*)$ is a monotonically increasing function, $\Omega_{\varepsilon}(t)$ is enclosed in an $n$-dimensional cube of constant size around the point $s=0$, each of whose sides has length

$$
L_{\varepsilon}(t)=2 \varepsilon\left\|g^{-1}[\Xi(t)]\right\|<2 \varepsilon\left\|g^{-1}(1)\right\|:=\Sigma
$$

This gives an estimate of the $n$-dimensional cubical region $\Omega_{\varepsilon}$ (each of whose sides is estimated to be of constant length $\Sigma$ ) to which trajectories of the controlled actual system will be attracted to.

Noting the fact that $\|s(t)\|$ is bounded by $L_{\varepsilon} / 2$ inside the surface $\Omega_{\varepsilon}$, an estimate of the error bounds is now given by

$$
\|\boldsymbol{e}(t)\| \leq \frac{\Sigma}{2 k} \quad \text { and } \quad\|\dot{e}(t)\| \leq \Sigma, \quad \text { as } t \rightarrow \infty
$$

Further, under the proviso $\|\overline{\mathbf{M}}(t)\|\|\dot{\boldsymbol{e}}(t)\| \ll 1$ for $t \in[0, \tau]$, in which $[0, \tau]$ is the interval over which the control is applied, which is something expected, it follows that

$$
L_{\varepsilon}(t)<\approx 2 \varepsilon\left\|g^{-1}\left[(\gamma(t)+k) /\left(\gamma(t)+\beta_{0}\right)\right]\right\|
$$

For ease of implementation, one could choose the function $\gamma(t)$ to be a constant by taking it to be the upper bound, $\gamma_{m}$, so that $\|\delta \ddot{\boldsymbol{q}}(t)\| \leq \gamma_{m}$ for $t \in[0, \tau]$. Then, Eq. (79) becomes

$$
L_{\varepsilon}<\approx 2 \varepsilon\left\|g^{-1}\left[\left(\gamma_{m}+k\right) /\left(\gamma_{m}+\beta_{0}\right)\right]\right\|
$$

One can then, accordingly, obtain an estimate of the error bounds by replacing $\Sigma$ in the expressions in Eq. (하) by the expression on the right-hand side of Eq. ( $\underline{80})$ :

$$
\|\boldsymbol{e}(t)\| \leq \frac{L_{\varepsilon}}{2 k} \quad \text { and } \quad\|\dot{e}(t)\| \leq L_{\varepsilon}, \quad \text { as } t \rightarrow \infty
$$

Main Result: The closed-form generalized sliding-surface controller for the uncertain system,

$$
\begin{aligned}
\mathbf{M}_{a} \ddot{\boldsymbol{q}}_{c}=\boldsymbol{Q}_{a}+\boldsymbol{Q}^{c}(t)+\mathbf{M} \ddot{\boldsymbol{u}}_{c} \\
\quad=\boldsymbol{Q}_{a}+\boldsymbol{Q}^{c}(t)-\mathbf{M}\left(k \dot{\boldsymbol{e}}+\left\{\frac{n\left[\gamma(t)+\beta_{0}\right]}{\alpha_{0}}\right\} \boldsymbol{f}(\boldsymbol{s})\right)
\end{aligned}
$$

in which the following conditions hold:

1) The control force $Q^{c}(t)$ is given by Eq. ( $)$, and is obtained on the basis of the nominal system.

2) The parameter $k>0$ is an arbitrary small positive number.

3) The function $f(s)$ is any arbitrary monotonically increasing odd continuous function of $s$ on the interval $(-\infty,+\infty)$, as described in Eq. (62) with $\|f(s)\| \geq 1$ outside $\Omega_{\varepsilon}$.

4) The norm $\|\delta \ddot{\boldsymbol{q}}(t)\| \leq \gamma(t)$ where $\gamma(t)$ is chosen based on the estimate of $\|\delta \ddot{\boldsymbol{q}}(t)\|$ from Eq. (53).

5) The parameter $\alpha_{0}$ is a small positive number that satisfies

$$
0<\alpha_{0}<(1-n\|\overline{\mathbf{M}}(t)\|)
$$

over the time duration over which the control is done, and under the proviso, and the expectation, that $\|\overline{\mathbf{M}}\|\|\dot{\boldsymbol{e}}\| \ll 1, \beta_{0}$ is chosen such that

$$
\beta_{0}=k
$$

will cause the actual system to track the trajectory of the nominal system within estimated error bounds Eq. (1).

Proof: Using Eq. (44) in Eq. (54), one can have

$$
\ddot{\boldsymbol{e}}=\ddot{\boldsymbol{q}}_{c}-\ddot{\boldsymbol{q}}=\delta \ddot{\boldsymbol{q}}+\mathbf{M}_{a}^{-1} \mathbf{M} \ddot{\boldsymbol{u}}_{c}
$$

so that

$$
\ddot{\boldsymbol{q}}_{c}=\ddot{\boldsymbol{q}}+\delta \ddot{\boldsymbol{q}}+\mathbf{M}_{a}^{-1} \mathbf{M} \ddot{\boldsymbol{u}}_{c}
$$

Consider Eq. (47):

$$
\begin{aligned}
\delta \ddot{\boldsymbol{q}} & =\left(\boldsymbol{a}_{a}-\boldsymbol{a}\right)+\left(\mathbf{M}_{a}^{-1}-\mathbf{M}^{-1}\right) \boldsymbol{Q}^{c}(t) \\
& =\left[\boldsymbol{a}_{a}+\mathbf{M}_{a}^{-1} \boldsymbol{Q}^{c}(t)\right]-\left[\boldsymbol{a}+\mathbf{M}^{-1} \boldsymbol{Q}^{c}(t)\right] \\
& =\boldsymbol{a}_{a}+\mathbf{M}_{a}^{-1} \boldsymbol{Q}^{c}(t)-\ddot{\boldsymbol{q}}
\end{aligned}
$$

In the last equality in Eq. (87), Eq. (9) has been used.

Substituting Eq. (87) in Eq. (6) yields

$$
\ddot{\boldsymbol{q}}_{c}=\boldsymbol{a}_{a}+\mathbf{M}_{a}^{-1} \boldsymbol{Q}^{c}(t)+\mathbf{M}_{a}^{-1} \mathbf{M} \ddot{\boldsymbol{u}}_{c}
$$

Premultiplying both sides of Eq. (요) by $\mathbf{M}_{a}$, one can obtain

$$
\mathbf{M}_{a} \ddot{\boldsymbol{q}}_{c}=\boldsymbol{Q}_{a}+\boldsymbol{Q}^{c}(t)+\mathbf{M} \ddot{\boldsymbol{u}}_{c}
$$

Finally, using Result [Eq. (6ㅜ)] and Eq. (1), the main result follows. 
When there is no uncertainty, the vectors $\boldsymbol{e}$ and $f(s)$ in Eq. (82) are identically zero, and no additional compensation for it is required.

\section{Numerical Results and Simulations for Attitude and Orbital Controls}

In this section, an example is introduced to demonstrate the applicability of the control methodology suggested in the previous sections. The numerical integration throughout this paper is done in the MATLAB environment, using a variable time step ode $15 \mathrm{~s}$ integrator with a relative error tolerance of $10^{-8}$ and an absolute error tolerance of $10^{-12}$.

Let us consider a system, in which there is only one follower satellite whose nominal mass is $m=120 \mathrm{~kg}$. Also, its nominal moments of inertia along its respective body-fixed axes are taken to be $\mathbf{J}=\operatorname{diag}\left(\begin{array}{lll}10 & 10 & 7.2\end{array}\right) \mathrm{k} \cdot \mathrm{gm}^{2}$. The value of $J_{0}$ has been arbitrarily chosen as $15 \mathrm{k} \cdot \mathrm{gm}^{2}$ [see Eq. (18)]. As previously assumed, the leader satellite is in an unperturbed circular orbit around a spherical Earth, and the radius of its orbit is $r_{L}=7 \times 10^{6} \mathrm{~m}$. For the sake of simplicity, the inclination $i_{L}$ of the leader satellite's orbit is taken to be $0 \mathrm{deg}$, that is, the leader satellite is just above the equator, and it is assumed that the leader satellite is on the $X$ axis of the ECI frame at the initial time $(t=0)$. The leader satellite's mean motion and orbital period are given by, respectively

$$
\begin{aligned}
& n_{L}=\sqrt{\frac{G M_{\oplus}}{r_{L}^{3}}}=1.0780 \times 10^{-3} \mathrm{rad} / \mathrm{s}, \\
& P_{L}=\frac{2 \pi}{n_{L}}=5.8285 \times 10^{3} \mathrm{~s}=1.6190 \mathrm{~h}
\end{aligned}
$$

The quantity $2 P_{L}$ (two orbital periods of the leader satellite) is chosen as the duration of time used for numerical integration, and the three orbital and attitude requirements [see Eq. (31)] are applied to the formation system, which are introduced in Sec. III. For the radius of PCO in Eq. (31a), $\rho_{0}=7.0 \times 10^{4} \mathrm{~m}$ is chosen, and the constant rotational frequency $\omega$ [see Eq. (31a)] is set to equal $n_{L}$ in Eq. (90).

The initial conditions chosen for orbital motion of the follower satellite are

$$
\begin{gathered}
x(0)=0 \mathrm{~m}, \quad y(0)=7.0 \times 10^{4} \mathrm{~m}, \quad z(0)=0 \mathrm{~m} \\
\dot{x}(0)=37.7347 \mathrm{~m} / \mathrm{s}, \quad \dot{y}(0)=0 \mathrm{~m} / \mathrm{s}, \quad \dot{z}(0)=75.4695 \mathrm{~m} / \mathrm{s}
\end{gathered}
$$

and the initial conditions for attitude motion as

$$
\begin{aligned}
& u_{0}(0)=0.0707372, \quad u_{1}(0)=0.997482, \quad u_{2}(0)=0.00498729, \\
& u_{3}(0)=3.536772 \times 10^{-4} \quad \dot{u}_{0}(0)=-0.00870185, \\
& \dot{u}_{1}(0)=6.143960 \times 10^{-4}, \quad \dot{u}_{2}(0)=5.403876 \times 10^{-4}, \quad \dot{u}_{3}(0)=0
\end{aligned}
$$

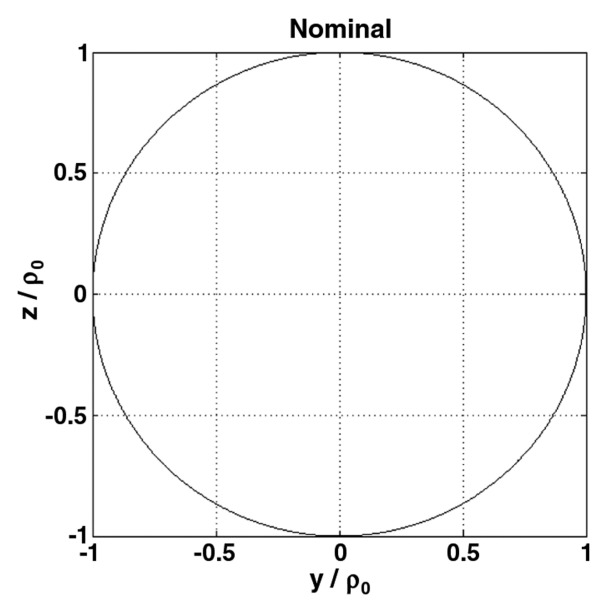

It must be noted that the initial conditions given by Eqs. (91) and (92) satisfy the trajectory requirements given in Eq. (31). The generalized control force required to satisfy these requirements is explicitly given by Eq. ( ). Application of this control force yields the trajectories of the motion of the follower.

Figure 1 represents the orbit of the follower satellite projected on the $y z$ plane (left) and $x z$ plane (right) in the Hill frame, respectively. This is the trajectory of the nominal system. The scale is normalized by $\rho_{0}$, and as seen in the figure, in these normalized coordinates, the follower satellite stays on a circle of radius unity around the leader satellite, which is located at the origin. In Fig. 2, the time history of each component of the quaternions for the follower satellite is shown, in which time is normalized by $P_{L}$, the period of the leader satellite [see Eq. (90)]. Figure 3 depicts the obtained control forces and their magnitude per unit mass of the follower satellite to follow the desired orbital requirements. The force components are described in the Hill frame, and time is normalized by $P_{L}$. Figure 4 illustrates the control torques and their magnitude per unit mass of the follower satellite for satisfying the attitude requirements (with no uncertainties). The torque components are described in the body frame of the follower satellite, and these physically applied torques are obtained using Eq. (30). Figure 5 represents errors in satisfying the desired nominal trajectories, assuming no uncertainties, described by Eq. (31). Instead of Eq. (31b), a new parameter $\theta$ is used, which is the angle between the $x$ axis of the body frame and the vector $\mathbf{P}\left[\begin{array}{lll}-X & -Y & -Z\end{array}\right]^{T}$ connecting the follower satellite and the center of Earth. These errors are denoted by 1) $\left.e_{1}(t)=2 x-z, 2\right) e_{2}(t)=y-\rho_{0} \cos (\omega t)$, 3) $\left.e_{3}(t)=z-\rho_{0} \sin (\omega t), 4\right) e_{4}(t)=\theta$, and 5) $e_{5}(t)=u_{0}^{2}+u_{1}^{2}+$ $u_{2}^{2}+u_{3}^{2}-1$.

To see how the response of the assumed nominal system can be altered through the effect of the uncertainty in the modeling process, consider, for reasons of simplicity, only the uncertainties in the mass $m$ of the follower satellite and in its moments of inertia $J_{x}, J_{y}$, and $J_{z}$. It is estimated that the actual values of these parameters differ from the nominal (best-estimate) values by a random uncertainty of $\pm 10 \%$ of the nominal values chosen. For illustrative purposes, a specific system with $\delta m=12, \delta J_{x}=1, \delta J_{y}=1$, and $\delta J_{z}=0.72$ is considered, and a simulation is again performed using Eq. (9), except that the best-estimate mass matrix of the uncontrolled system [see Eq. (1)] is replaced with the actual mass matrix $\mathbf{M}_{a}:=\mathbf{M}+\boldsymbol{\delta} \mathbf{M}$, in which $\mathbf{M}$ is defined in Eq. (29), with all other parameter values the same as previously prescribed. It is noted that the elements of the $7 \times 7$ symmetric matrix $\mathbf{M}_{a}$ are given in a manner similar to Eq. (29). In this case, $m$ and $J_{i}$ in Eq. (29) are replaced with $m=m+\delta m$ and $J_{i}=J_{i}+\delta J_{i}$, respectively. Using the control forces and torque obtained under the assumption that the mass and moments of inertia are those of the nominal system $\left[\boldsymbol{Q}^{c}(t)\right]$, one can obtain

$$
\ddot{\tilde{\boldsymbol{q}}}:=\mathbf{M}_{a}^{-1} \boldsymbol{Q}(\tilde{\boldsymbol{q}}, \dot{\tilde{\boldsymbol{q}}}, t)+\mathbf{M}_{a}^{-1} \boldsymbol{Q}^{c}(t)
$$

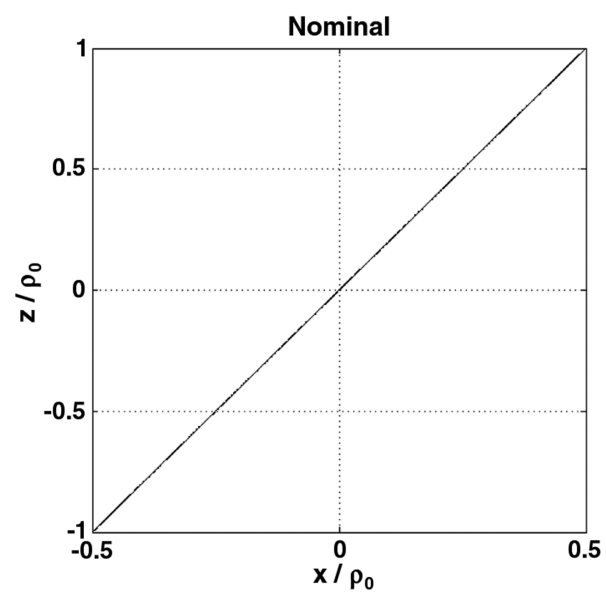

Fig. 1 Constrained motion of the nominal system with no uncertainties assumed. 


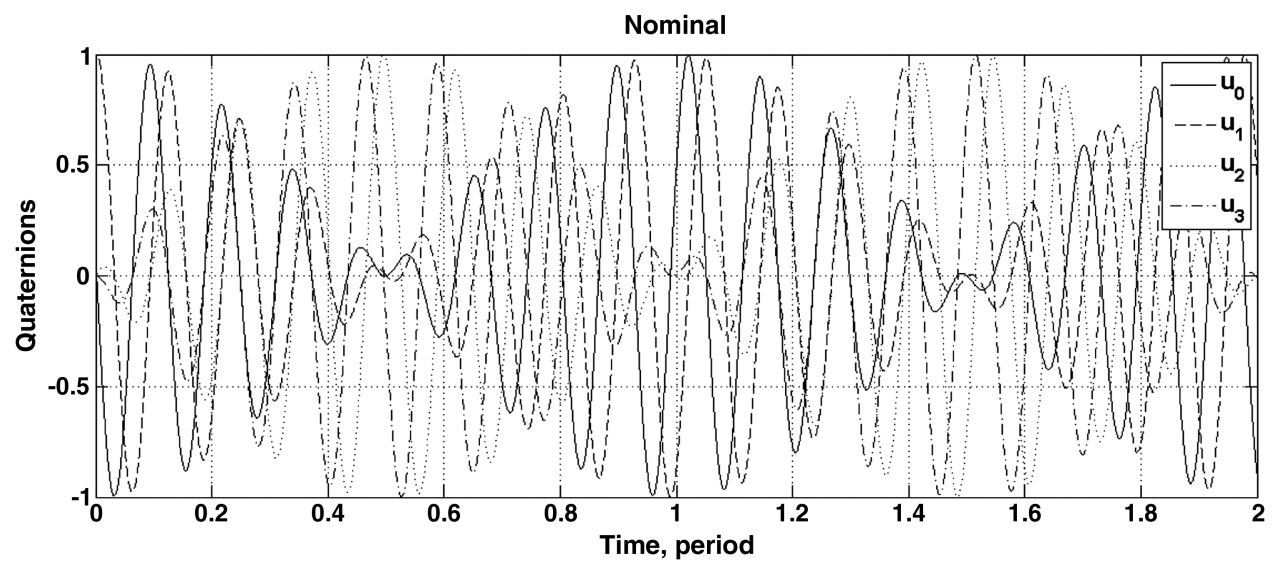

Fig. 2 Time history of quaternions of the nominal system with no uncertainties assumed.
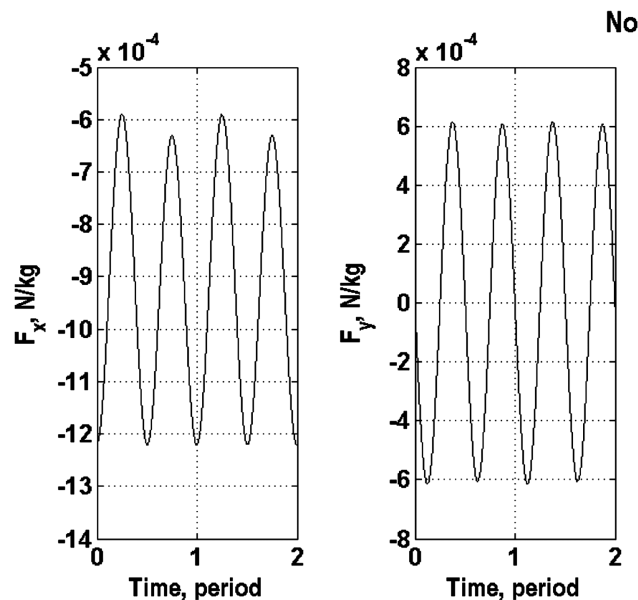

Nominal

Fig. 3 Required control forces to satisfy the nominal orbital constraints.

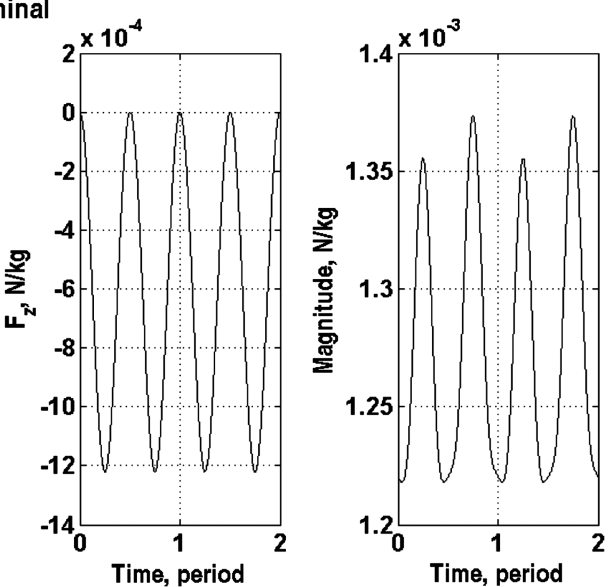

The trajectories $(\tilde{\boldsymbol{q}}, \dot{\tilde{\boldsymbol{q}}})$ of the system Eq. $(\underline{93})$ with the actual mass and moments of inertia are determined.

Figure $\underline{6}$ shows these orbital trajectories of the actual system projected on the $y z$ plane (left) and $x z$ plane (right) in the Hill frame, respectively. Figure 7 depicts the time history of quaternions of the actual uncertain system. Both figures are different from those obtained from the nominal system, showing that a misassessment of the mass and moments of inertia of the follower satellite can have significant consequences. The resulting quaternions satisfy neither the Earth-pointing constraint nor the unit-norm constraint.

The structure and parameters for the continuous controller $\ddot{\boldsymbol{u}}_{c}$ given by Eq. (64) are chosen as

$$
f_{i}(s)=\left(s_{i} / \varepsilon\right)^{3}
$$

in which $\varepsilon>0$ is a suitable small number. Thus, the closed-form additional controller needed to compensate for uncertainties in the actual system is obtained as

$$
\ddot{\boldsymbol{u}}_{c}(t)=-k \dot{\boldsymbol{e}}-\left\{\frac{n\left[\gamma(t)+\beta_{0}\right]}{\alpha_{0}}\right\}(s / \varepsilon)^{3}
$$

It is noted that, with this choice of $f_{i}(s)=\left(s_{i} / \varepsilon\right)^{3}$, the region outside the surface $\Omega_{\varepsilon}$ is the region outside of the $n$-dimensional cube around $s=0$, each of whose sides has length $L_{\varepsilon}<\approx 2 \varepsilon\left[\left(\gamma_{m}+k\right) /\right.$
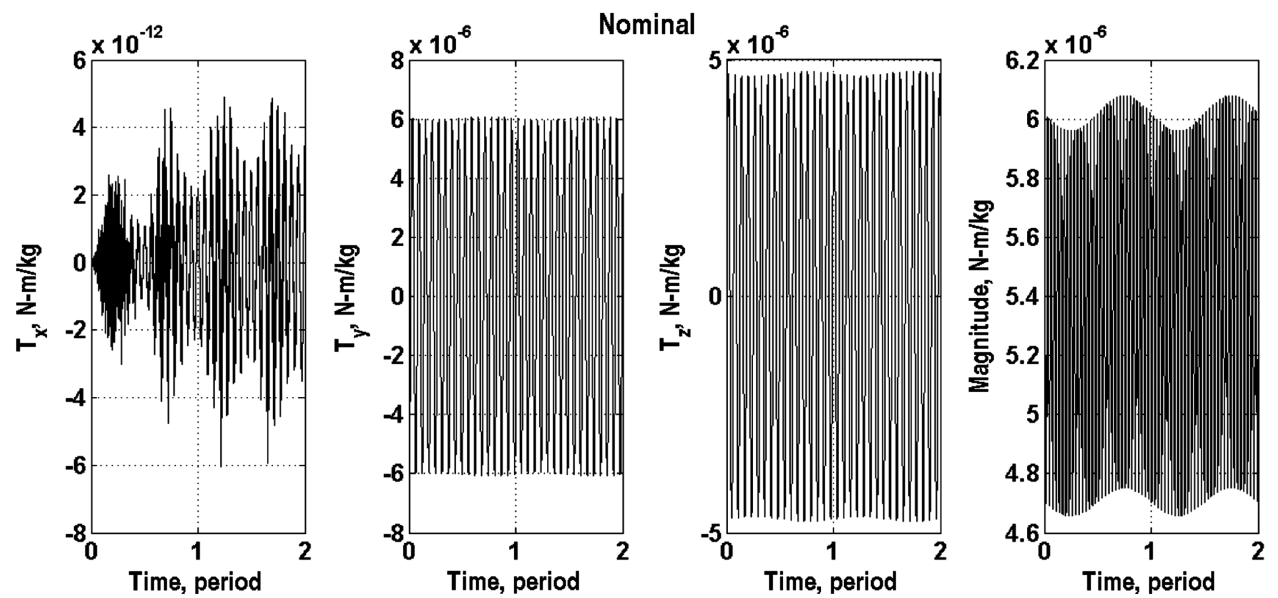

Fig. 4 Required control torques to satisfy the nominal attitude constraints. 


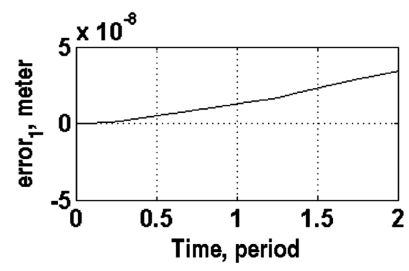

a)

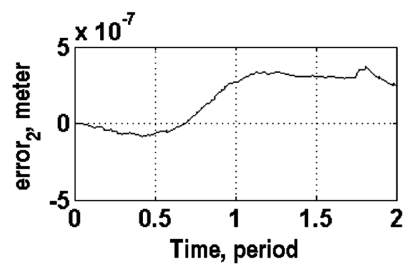

b)

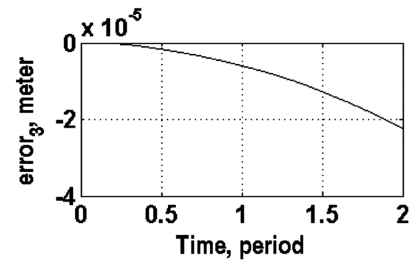

c)

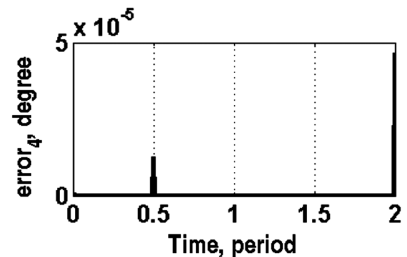

d)

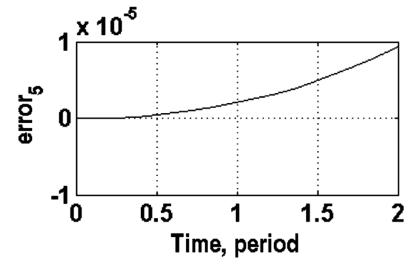

e)

Fig. 5 Errors in the satisfaction with the nominal constraints.
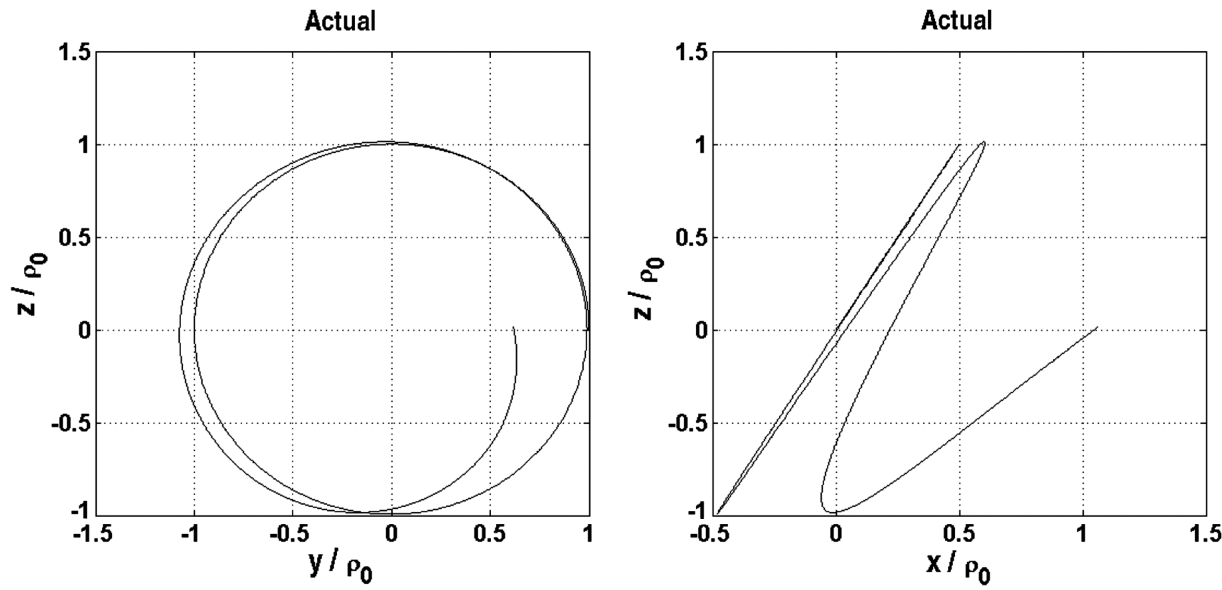

Fig. 6 Motion of the actual uncertain system.

$\left.\left(\gamma_{m}+\beta_{0}\right)\right]^{1 / 3}$ [see Eq. (80)]. In this region, Eq. (74) assures that the control given by Eq. (95) will cause $s(t)$ to strictly decrease, until it reaches the boundary $s \in \Omega_{\varepsilon}$ and remains inside this $n$ box thereafter.

Premultiplying both sides of Eq. ( $\underline{82})$ by $\mathbf{M}_{a}^{-1}$ and using the additional controller Eq. (95), one can obtain the closed-form equation of motion of the controlled actual system as

$$
\ddot{\boldsymbol{q}}_{c}=\boldsymbol{a}_{a}+\mathbf{M}_{a}^{-1} \boldsymbol{Q}^{c}(t)-\mathbf{M}_{a}^{-1} \mathbf{M}\left(k \dot{\boldsymbol{e}}+\left\{\frac{n\left[\gamma(t)+\beta_{0}\right]}{\alpha_{0}}\right\}(\boldsymbol{s} / \varepsilon)^{1 / 3}\right)
$$

which will cause the actual system to track the trajectory of the nominal system, thereby compensating for the uncertainty in the knowledge of the actual system. When there is no uncertainty, the vectors $\boldsymbol{e}$ and $\boldsymbol{s}$ go to zero, and $\mathbf{M}_{a}=\mathbf{M}$ giving relation Eq. (9).

With the knowledge that there is a $\pm 10 \%$ uncertainty in the mass and moments of inertia of the follower satellite, the norm of Eq. (52) is used to estimate $\gamma(t)$ and $\gamma_{m}\left[\gamma(t) \leq \gamma_{m}\right]$. For the simulation, the parameters are chosen as $n=7, \gamma(t)=\gamma_{m}=10^{-2}, \beta_{0}=0.1$, $k=0.1, \alpha_{0}=0.5$, and $\varepsilon=10^{-4}$. It should be noted that the estimate of $\gamma(t)$ is not sensitive to the magnitude of additional control forces $Q^{u}$ in the control approach.

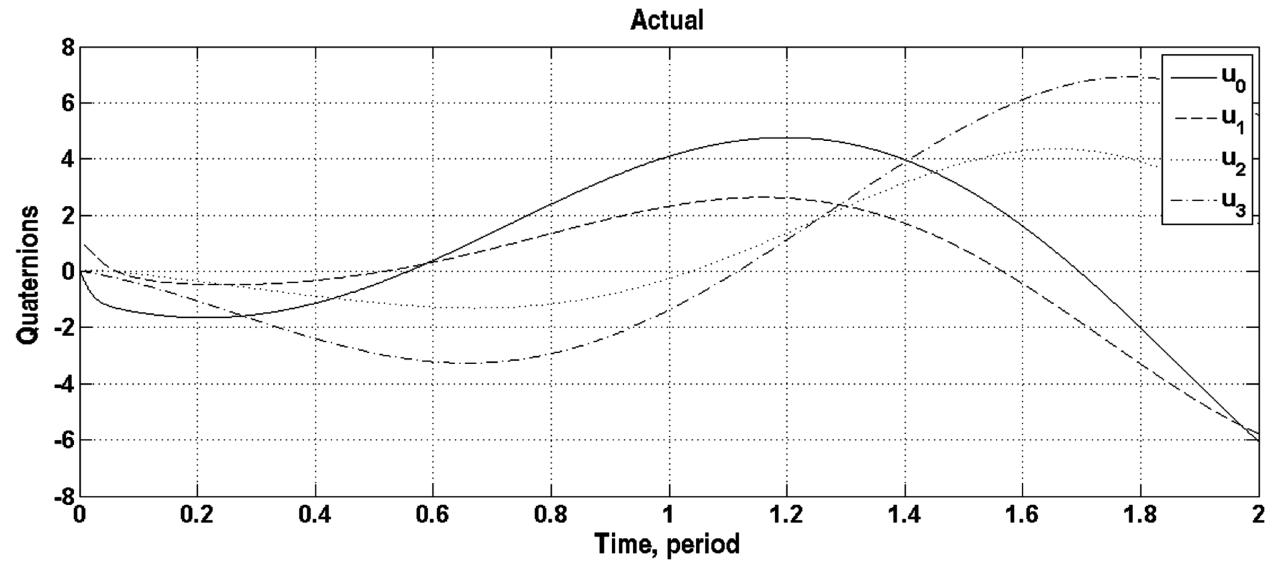

Fig. 7 Quaternions of actual uncertain follower satellite with no uncertainty compensation. 


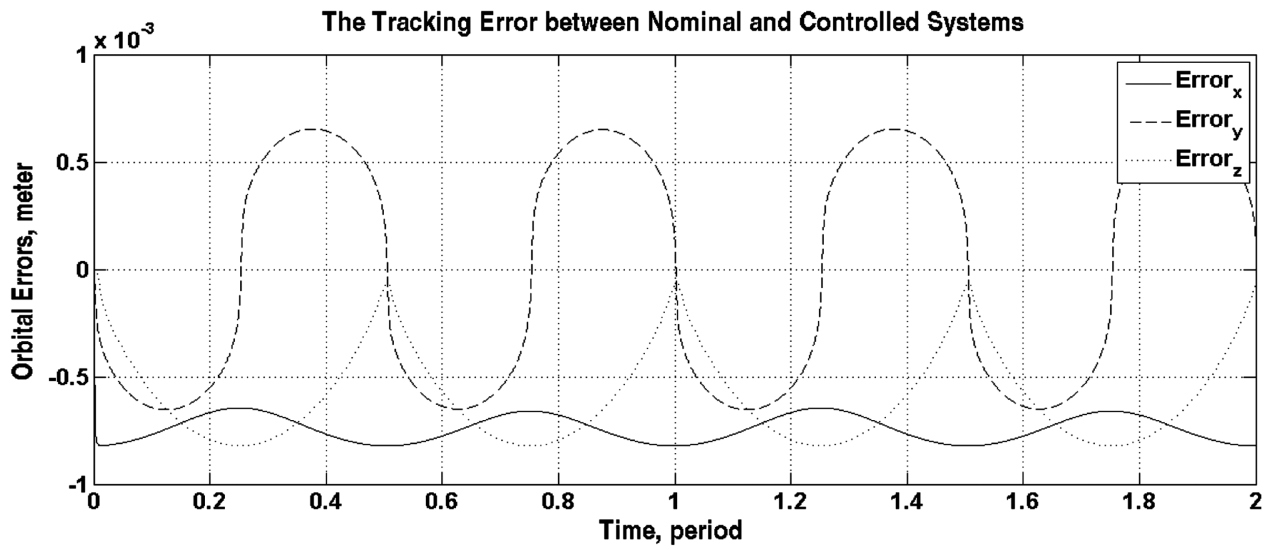

Fig. 8 Orbital errors between the nominal and controlled systems.

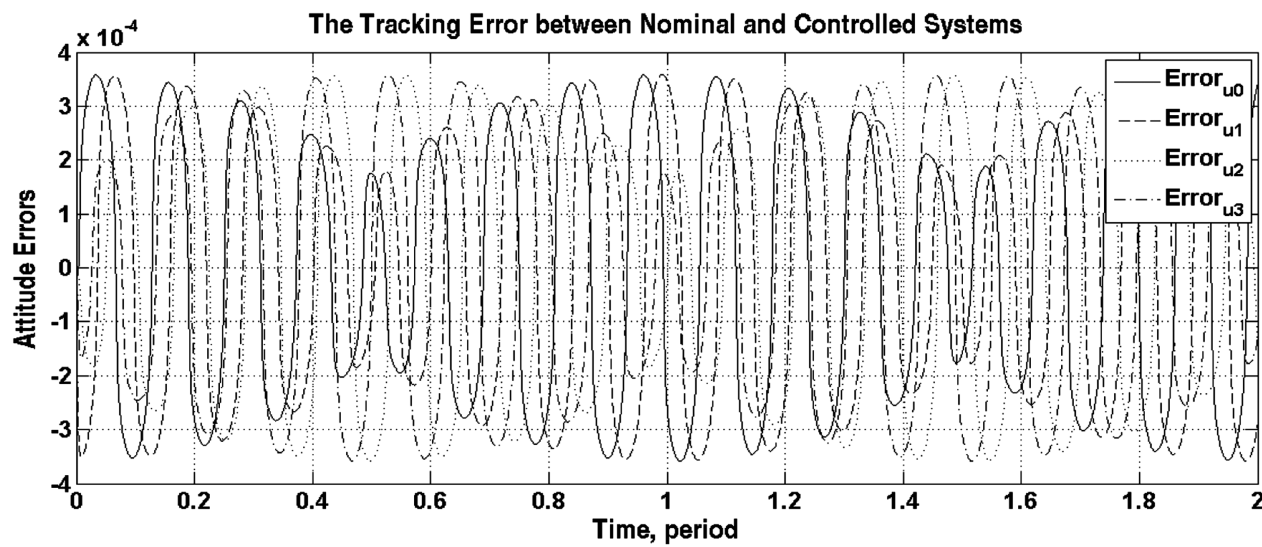

Fig. 9 Attitude (quaternion) errors between the nominal and controlled systems.
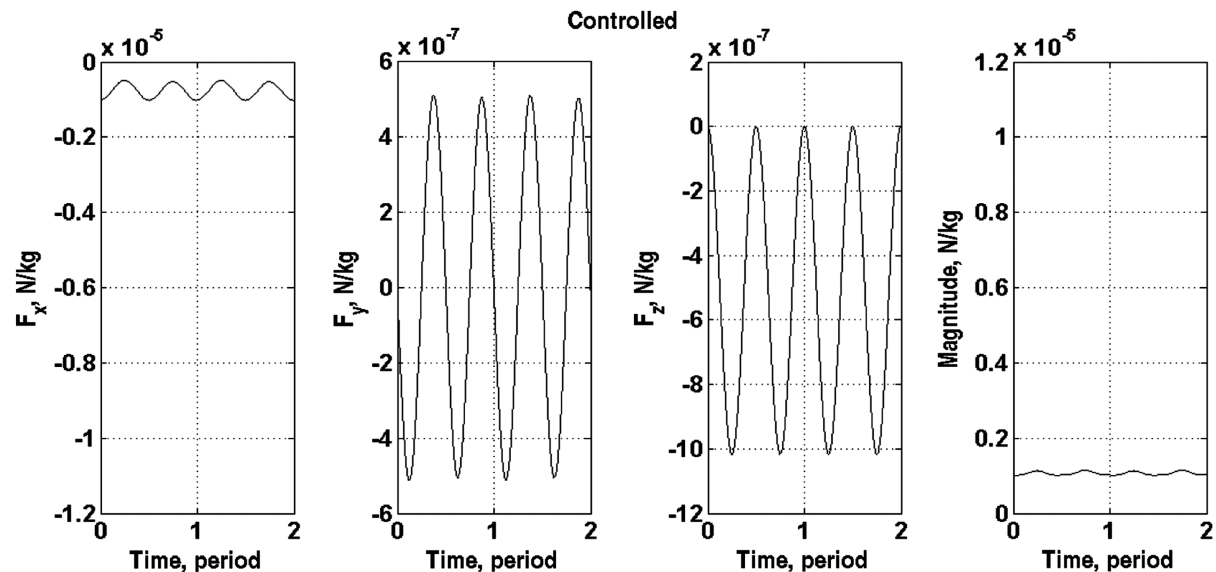

Fig. 10 Required additional control force to compensate for uncertainties in follower satellite.
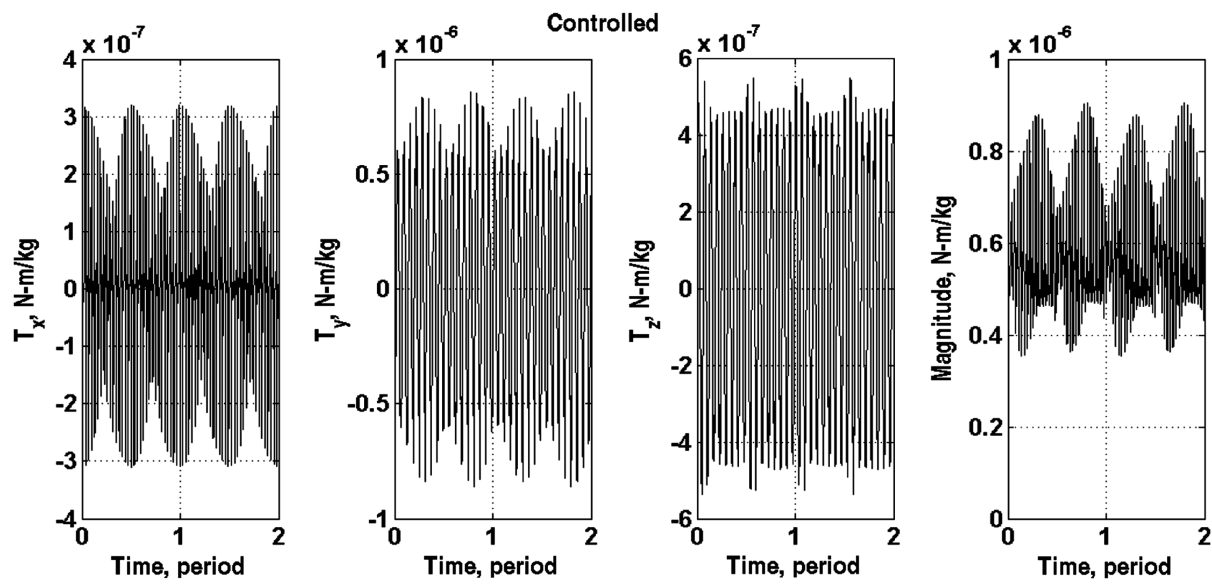

Fig. 11 Required additional torque to compensate for uncertainties in follower satellite. 
At the scales shown, the controlled trajectories of the follower satellite projected on the $y z$ plane and $x z$ plane in the Hill frame fall exactly on those shown in Fig. 1 . The errors in tracking the attitude and the orbital trajectories of the nominal system are shown in Figs. $\underline{8}$ and 9. In Fig. 8, one can see that there are minute differences between the position coordinates of the nominal and the controlled trajectories of the follower satellite, and the attitude differences, as represented by the quaternion vectors, are also small, as seen in Fig. 9. Thus, the uncertain system can be controlled to behave in exactly the same way the nominal (best-estimate) system is desired to behave.

Figures 10 and 11 , respectively, show the additional control forces and torques, as well as their magnitudes per unit mass of the follower satellite to compensate for the uncertainties assumed. Both additional control forces and torques are seen to be small when compared with those obtained from the nominal system (Figs. $\underline{3}$ and $\underline{4}$, respectively).

In the current example, Eq. (으) yields

$$
L_{\varepsilon}<\approx 2 \varepsilon\left[\left(\gamma_{m}+k\right) /\left(\gamma_{m}+\beta_{0}\right)\right]^{1 / 3} \approx 2 \times 10^{-4}
$$

so that the calculated error-norm estimate is $\|\boldsymbol{e}(t)\|<\approx L_{\varepsilon} / 2 k \approx$ $10^{-3}$ [see Eq. (81)]. Figures 8 and 9 show that the errors are within the estimated error norm. It is also noted that the use of the specified smooth cubic function eliminates chattering.

\section{Conclusions}

In this paper, a simple method for the formation-keeping problem with attitude and orbital requirements, in the presence of model uncertainties, has been developed. The closed-form nonlinear controller developed herein yields the results that can be used for controlling a satellite formation, in which each satellite needs to satisfy attitude and orbital requirements in the presence of uncertainties with known bounds. The approach relies on 1) the determination of the closed-form control of the nonlinear nominal system that will permit exact trajectory tracking, and 2) the development of a continuous set of controllers that have no chattering and that can allow the trajectory of the nominal system to be tracked with preassigned accuracy. It is illustrated by considering a follower satellite whose mass and moments of inertia are uncertain. The numerical simulation shows the simplicity and accuracy of the approach developed herein.

Because the control force and torque to be applied to the follower satellites are explicitly obtained in closed form, and the method is not computationally intensive, it can be easily used for on-orbit real-time control of maneuvers, especially for formations with many satellites, for which the underlying dynamics are highly nonlinear. Also, the control function $f_{i}(s)$ and the parameters that define the compensating controller can be chosen, depending on a practical consideration of the control environment, and on the extent to which the compensation of uncertainties is desired. These parameters can be adjusted so that desired error bounds can be guaranteed when the uncertain system is required to track the nominal system. For example, the use of a cubic function may obviate the need for a highgain controller. Furthermore, because the control is continuous, chattering is prevented. For brevity, only uncertainties that are related to the mass and moments of inertia of the follower satellite have been illustrated in the numerical example. However, the formulation of the current methodology encompasses both general sources of uncertainties - uncertainties in the description of the physical system and uncertainties in knowledge of the generalized given forces applied to the system. The closed-form controller developed herein is therefore general enough to be applicable to complex dynamic system of multisatellites, in which the uncertainties in the given forces may be important.

\section{References}

[1] Aoude, G. S., How, J. P., and Garcia, I. M., "Two-Stage Path Planning Approach for Designing Multiple Spacecraft Reconfiguration Maneuvers," Proceedings of the 20th International Symposium on Space Flight Dynamics, NASA CP-2007-214158, Greenbelt, MD, 2007, pp. 1-16.
[2] Hill, G. W., "Researches in the Lunar Theory," American Journal of Mathematics, Vol. 1, No. 1, 1878, pp. 5-26. doi: $10.2307 / 2369430$

[3] Clohessy, W. H., and Wiltshire, R. S., "Terminal Guidance System for Satellite Rendezvous," Journal of the Aerospace Sciences, Vol. 27, No. 8, 1960, pp. 653-658.

[4] Tschauner, J., and Hempel, P., "Rendezvous zu Einemin Elliptischer Bahn Umlaufenden Ziel," Acta Astronautica, Vol. 11, No. 2, 1965, pp. 104-109.

[5] Yan, Q., Kapila, V., and Sparks, A. G., "Pulse-Based Periodic Control for Spacecraft Formation Flying," Proceeding of American Control Conference, IEEE Publ., Piscataway, NJ, 2000, pp. 374-378.

[6] Won, C. H., and Ahn, H. S., "Nonlinear Orbital Dynamic Equations and State-Dependent Riccati Equation Control of Formation Flying Satellites," Journal of the Astronautical Sciences, Vol. 51, No. 4, 2003, pp. 433-449.

[7] Long, M. R., and Hall, C. D., "Attitude Tracking Control for Spacecraft Formation Flying," Proceedings of the 1999 Flight Mechanics Symposium, NASA CP-1999-209235, Greenbelt, MD, May 1999, pp. 319-332.

[8] Ahn, C., and Kim, Y., "Point Targeting of Multisatellites via a Virtual Structure Formation Flight Scheme," Journal of Guidance, Control, and Dynamics, Vol. 32, No. 4, 2009, pp. 1330-1344.

[9] Lee, K. W., and Singh, S. N., "Variable Structure Model Reference Adaptive Formation Control of Satellites," Journal of Guidance, Control, and Dynamics, Vol. 35, No. 1, 2012, pp. 104-115. doi: $10.2514 / 1.53904$

[10] Godard, G., and Kumar, K. D., "Fault Tolerant Reconfigurable Satellite Formations Using Adaptive Variable Structure Techniques," Journal of Guidance, Control, and Dynamics, Vol. 33, No. 3, 2010, pp. 969-984. doi: $10.2514 / 1.38580$

[11] Udwadia, F. E., "Equations of Motion for Mechanical Systems: A Unified Approach," International Journal of Non-Linear Mechanics, Vol. 31, No. 6, 1996, pp. 951-958. doi:10.1016/S0020-7462(96)00116-3

[12] Udwadia, F. E., "Nonideal Constraints and Lagrangian Dynamics," Journal of Aerospace Engineering, Vol. 13, No. 1, 2000, pp. 17-22. doi:10.1061/(ASCE)0893-1321(2000)13:1(17)

[13] Udwadia, F. E., "A New Perspective on the Tracking Control of Nonlinear Structural and Mechanical Systems," Proceedings of the Royal Society of London, Series A: Mathematical and Physical Sciences, Vol. 459, No. 2035, 2003, pp. 1783-1800. doi:10.1098/rspa.2002.1062

[14] Peters, J., Mistry, M., Udwadia, F. E., and Schaal, S., "A Unifying Framework for Robot Control with Redundant DOFs," Autonomous Robots, Vol. 24, May 2008, pp. 1-12. doi:10.1007/s10514-007-9051-x

[15] Udwadia, F. E., "Optimal Tracking Control of Nonlinear Dynamical Systems," Proceedings of the Royal Society of London, Series A: Mathematical and Physical Sciences, Vol. 464, No. 2097, 2008, pp. 2341-2363. doi:10.1098/rspa.2008.0040

[16] Schutte, A., and Udwadia, F. E., "New Approach to the Modeling of Complex Multi-Body Dynamical Systems," Journal of Applied Mechanics, Vol. 78, No. 2, 2011, Paper 021018. doi:10.1115/1.4002329

[17] Cho, H., and Yu, A., "New Approach to Satellite Formation-Keeping: Exact Solution to the Full Nonlinear Problem," Journal of Aerospace Engineering, Vol. 22, No. 4, 2009, pp. 445-455. doi:10.1061/(ASCE)AS.1943-5525.0000013

[18] Udwadia, F. E., Schutte, A. D., and Lam, T., "Formation Flight of Multiple Rigid Body Spacecraft," 48th AIAA/ASME/ASCE/AHS/ASC Structures, Structural Dynamics, and Materials Conference, AIAA, Reston, VA, 2007, pp. 272-290; also AIAA Paper 2007-2391, 2007.

[19] Cho, H., and Udwadia, F. E., "Explicit Control Force and Torque Determination for Satellite Formation-Keeping with Attitude Requirements," Journal of Guidance, Control, and Dynamics, Vol. 36, No. 2, 2013, pp. 589-605. doi: $10.2514 / 1.55873$

[20] Vallado, D. A., Fundamentals of Astrodynamics and Applications, 2nd ed., The Space Technology Library, Microcosm, Hawthorne, CA, 2001, pp. 20-23, 157-158.

[21] Cho, H., and Udwadia, F. E., "Explicit Solution to the Full Nonlinear Problem for Satellite Formation-Keeping," Acta Astronautica, Vol. 67, Nos. 3-4, 2010, pp. 369-387. doi:10.1016/j.actaastro.2010.02.010

[22] Udwadia, F. E., and Schutte, A. D., "An Alternative Derivation of the Quaternion Equations of Motion for Rigid-Body Rotational Dynamics," Journal of Applied Mechanics, Vol. 77, No. 4, 2010, 
Paper 044505

doi: $10.1115 / 1.4000917$

[23] Sabol, C., Burns, R., and McLaughlin, C. A., "Satellite Formation Flying Design and Evolution," Journal of Spacecraft and Rockets, Vol. 38, No. 2, 2001, pp. 270-278. doi: $10.2514 / 2.3681$

[24] Henderson, H. V., and Searle, S. R., "On Deriving the Inverse of a Sum of Matrices," SIAM Review, Vol. 23, No. 1, 1981, pp. 53-60. doi: $10.1137 / 1023004$

[25] Utkin, V. I., "Variable Structure Systems with Sliding Mode," IEEE Transactions on Automatic Control, Vol. 22, No. 2, 1977, pp. 212-222.

doi:10.1109/TAC.1977.1101446

[26] Tsypkin, Y. Z., Teoriya Releinykh System Avtomati Cheskogo Regulirovaniya (Theory of Switching Control Systems), Gostekhizdat, Moscow, 1995, pp. 27-84.

[27] Edwards, C., and Spurgeon, S., Sliding Mode Control: Theory and Applications, Taylor and Francis, London, 1999, pp. 17-74.

[28] Khalil, H. K., Nonlinear Systems, Prentice-Hall, Upper Saddle River, NJ, 2002, pp. 551-589. 